DTNSRDC-86/054 September 1986

Ship Performance Department

Research and Development Report

\title{
Far-Field Features of the Kelvin Wake
}

by
A. Barnell
F. Noblesse
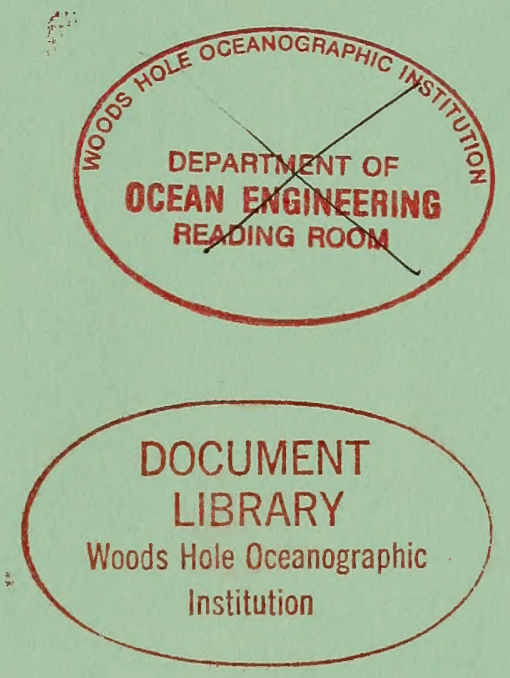


\section{MAJOR DTNSRDC TECHNICAL COMPONENTS}

\begin{tabular}{|c|c|c|c|}
\hline \multicolumn{4}{|c|}{ COMMANDER } \\
\hline $\mathrm{TECH}$ & NIC & CTO & A 01 \\
\hline $\begin{array}{r}\text { OFFICER IN CHARGE } \\
\text { CARDEROCK } \\
\end{array}$ & 05 & 04 & $\begin{array}{l}\text { OFFICER IN CHARGE } \\
\text { ANNAPOLIS }\end{array}$ \\
\hline $\begin{array}{r}\text { SHIP SYSTEMS INTEGRATION } \\
\text { DEPARTMENT }\end{array}$ & 12 & 27 & $\begin{array}{l}\text { PROPULSION AND AUXILIARY } \\
\text { SYSTEMS DEPARTMENT }\end{array}$ \\
\hline $\begin{array}{r}\text { SHIP PERFORMANCE } \\
\text { DEPARTMENT }\end{array}$ & 15 & 28 & $\begin{array}{l}\text { SHIP MATERIALS ENGINEERING } \\
\text { DEPARTMENT }\end{array}$ \\
\hline $\begin{array}{l}\text { AVIATION AND SURFACE } \\
\text { EFFECTS DEPARTMENT }\end{array}$ & 16 & & \\
\hline STRUCTURES DEPARTMENT & 17 & & \\
\hline $\begin{array}{r}\text { COMPUTATION, MATHEMATICS } \\
\& \text { LOGISTICS DEPARTMENT }\end{array}$ & 18 & & \\
\hline SHIP ACOUSTICS DEPARTMENT & 19 & & \\
\hline $\begin{array}{r}\text { CENTRAL INSTRUMENTATION } \\
\text { DEPARTMENT }\end{array}$ & 29 & & \\
\hline
\end{tabular}

DESTRUCTION NOTICE - For classified documents, follow the procedures in DOD 5220.22M, Industrial Security Manual, Section II-9, or DOD 5200.1-R, Information Security Program Regulation, Chapter IX. For unclassified, limited documents, destroy by any method that will prevent disclosure of contents or reconstruction of the document. 


\section{REPORT DOCUMENTATION PAGE}

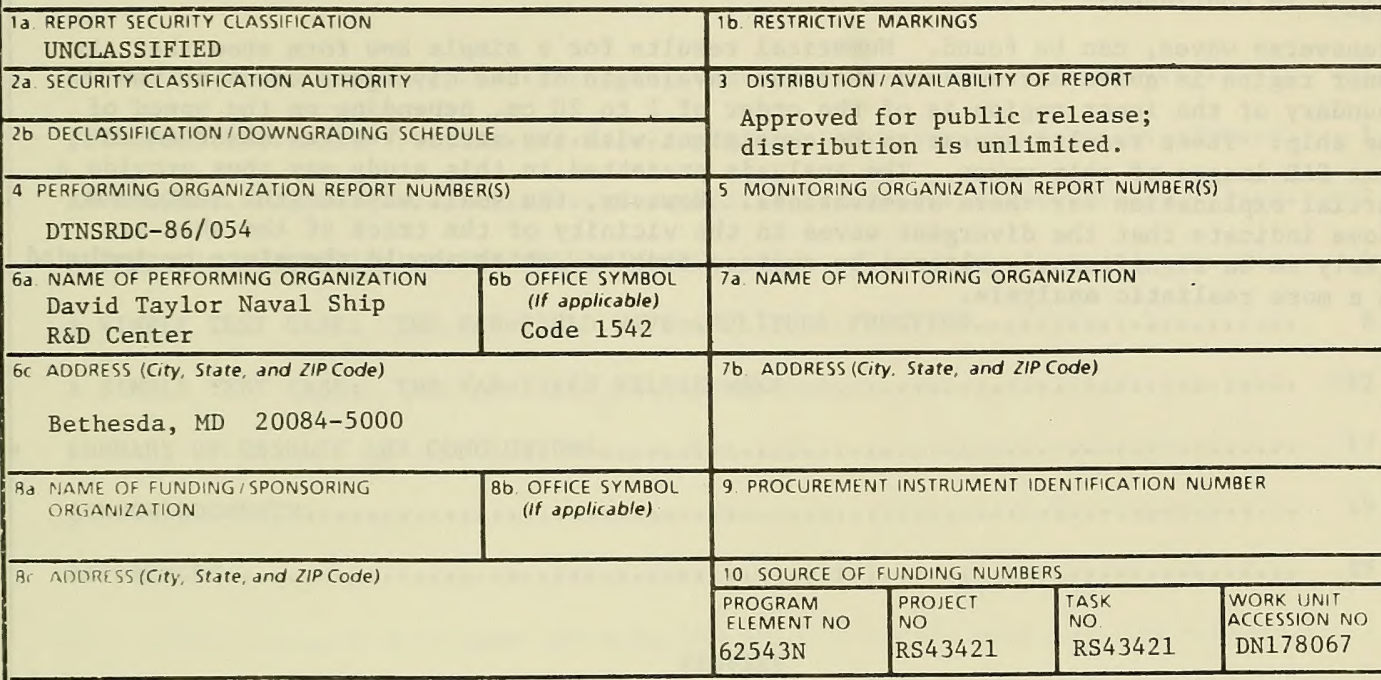

11 TITLE (Include Security Classification)

Far-Field Features of the Kelvin Wake

PFRSONAL AUTHOR(S)

Barnel1, A. and Noblesse, F.

\begin{tabular}{|l|l|l|l|l}
\hline $\begin{array}{l}\text { 13a TYPE UF REPORT } \\
\text { Final }\end{array}$ & $\begin{array}{l}\text { 13b TIME COVERED } \\
\text { FROM }\end{array}$ & $\begin{array}{l}\text { DATE OF REPORT (Year, MONth, Day) } \\
1986, \text { September }\end{array}$ & $\begin{array}{c}15 \text { PAGE COUNT } \\
33\end{array}$ \\
\hline
\end{tabular}

6 SUPPIEMENTARY NOTATION

\begin{tabular}{|c|c|c|c|}
\hline \multicolumn{3}{|c|}{ COSATI CODES } & \multirow{3}{*}{$\begin{array}{l}18 \text { SUBIECT TERMS (Continue on reverse if necessary and identify by block number) } \\
\text { Ship Waves } \\
\text { Kelvin Wake }\end{array}$} \\
\hline FIELD & GROUP & SUB-GROUP & \\
\hline & & & \\
\hline
\end{tabular}

3 ABSTRACT (Continue on reverse if necessary and identify by block number)

The classical Kelvin wake trailing a ship advancing at constant speed in calm water is studied. In particular, asymptotic expressions for determining the far-field wake, at large distances behind the ship, are investigated numerically. This analysis, in which surface tension and nonlinearities are ignored, indicates that the divergent waves of a surface ship generally are infinitely steep at the track of the ship, even though their amplitude vanishes there; this result is theoretically possible because the wavelength of the divergent waves vanishes at the track of the ship. Inasmuch as infinitely-steep water waves cannot exist in reality, the linear no-surface-tension analysis presented in this study suggests that no divergent waves can exist within a certain region in the vicinity of the track of the ship, and that the Kelvin wake contains three distinct regions: (i) an inner region where only transverse waves can exist, (ii) an outer region where both transverse and divergent waves are present, and (iii) an intermediate region at the boundary between the inner and outer regions where short steep divergent waves, as well as

(Continued)

\begin{tabular}{|c|c|c|c|}
\hline $\begin{array}{l}20 \text { DISTRIBUTION/AVAILABILITY OF ABSTRACI } \\
\text { MIINCLASSIFIEDIUNLIMITED } \square \text { SAME AS RPT }\end{array}$ & $\square$ DTIC USERS & $\begin{array}{l}21 \text { ABSTRACT SECURITY CLASSIFICAT } \\
\text { UNCLASSIFIED. }\end{array}$ & $N$ \\
\hline $\begin{array}{l}\text { NAME DF RESPONSIBLE INDIV } \\
\text { Alexander Barnel1 }\end{array}$ & & $\begin{array}{l}\text { 22b TELEPHONE (Include Area Code) } \\
(301) 227-1865\end{array}$ & $\begin{array}{l}\text { 22C OFFICE SYMBOI } \\
\text { Code } 1542\end{array}$ \\
\hline
\end{tabular}




\section{(Block 19 continued)}

transverse waves, can be found. Numerical results for a simple bow form show that the inner region is quite narrow, and that the wavelength of the divergent waves at the boundary of the inner region is of the order of 1 to $20 \mathrm{~cm}$, depending on the speed of the ship. These results appear to be consistent with the narrow $V$-wakes observed in some SAR images of ship wakes. The analysis presented in this study may thus provide a partial explanation for these observations. However, the small wavelengths mentioned above indicate that the divergent waves in the vicinity of the track of the ship are likely to be significantly altered by surface tension, which should therefore be included in a more realistic analysis. 


\section{CONTENTS}

Page

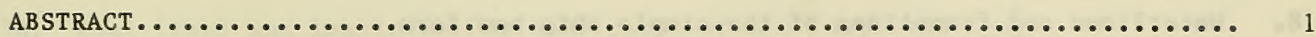

inTRODUCTION. . . . . . . . . . . . . . . . . . . . . . . . . . . . . . . . . . .

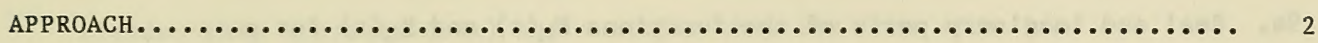

ASYMPTOTIC EVALUATION OF THE KELVIN WAKE.......................... 4

A Simple test CASE: The FAR-Field WAVE-Amplitude FunCtion............. 8

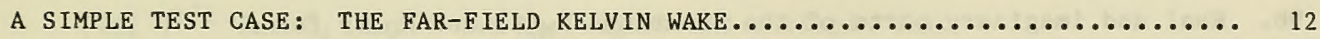

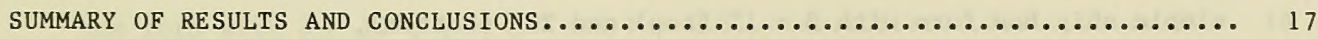

ACKNOWLEDGMENTS. . . . . . . . . . . . . . . . . . . . . . . . . . . . . . . . 19

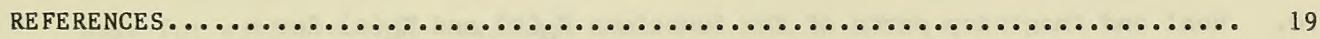

FIGURES

1. Real parts of the functions $E_{+}(t ; x, \alpha)$ and $E_{-}(t ; x, \alpha)$ for $x=-50$ and

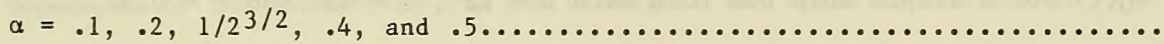

2. Real and imaginary parts of the functions $\left(1+t^{2}\right) K(t)$ and $t\left(1+t^{2}\right) K(t)$

for a simple ship bow shape in the zeroth-order slender-ship

approximation $\ldots \ldots \ldots \ldots \ldots \ldots \ldots \ldots \ldots \ldots \ldots \ldots \ldots \ldots \ldots \ldots \ldots \ldots \ldots \ldots \ldots \ldots \ldots \ldots$

3a. The phase function $\theta(t ; \alpha)$ for $0 \leqslant t \leqslant 6$ and several values of $\alpha \ldots \ldots \ldots$

3b. The function $\theta^{\prime}(t ; \alpha)$ for $0 \leqslant t \leqslant 6$ and several values of $\alpha \ldots \ldots \ldots \ldots$

3c. The function $\theta^{\prime \prime}(t ; \alpha)$ for $0 \leqslant t \leqslant 3$ and several values of $\alpha \ldots \ldots \ldots$

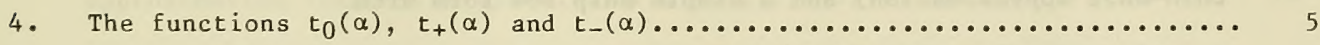

5. The functions $t_{0}(\alpha), t_{ \pm}(\alpha), \theta_{0}^{\prime}(\alpha), \theta_{0}^{\prime \prime \prime}(\alpha), \theta_{ \pm}^{\prime \prime}(\alpha)$

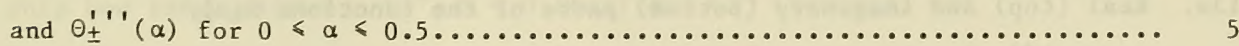

6. The classical Kelvin ship wave pattern........................

7. The wavelengths $\lambda_{ \pm}(\alpha)$ and propagation angles $\beta_{ \pm}(\alpha)$ of the transverse and divergent waves in the Kelvin wake........................... 
FIGURES (Continued)

Page

8. Waterlines and framelines of the simple ship bow form

considered for numerical applications.

9a. Real and imaginary parts of the functions $K_{M}(t)$ and $K_{0}(t)$ for a simple ship bow form with $B=12^{\circ}$ (top) and $3^{\circ}$ (bottom),

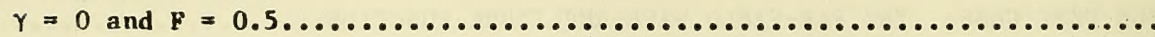

9b. Real and imaginary parts of the functions $K_{M}(t)$ and $K_{0}(t)$ for a simple ship bow form with $\beta=1^{\circ}$ (top) and $20^{\prime}$ (bottom), $\gamma=0$ and $\mathbf{F}=0.5$

10. Real and imaginary parts of the functions $K_{M}(t)$ and $K_{0}(t)$ for a stmple ship bow form with $Y=0$ and $45^{\circ}, B=12^{\circ}$ and $F=0.5 \ldots \ldots \ldots \ldots$

11. Real (top) and imaginary (bottom) parts of the functions $K_{M}(t)$ and

$K_{0}(t)$ for a simple ship bow form with $B=12^{\circ}, Y=45^{\circ}$ and $F=0.5 \ldots \ldots$

12a. Real (top) and imaginary (bottom) parts of the functions $K_{ \pm} a \frac{t}{k} /$

$\left(\mp \theta_{ \pm}^{\prime}\right)^{1 / 2}$ for $k=0$ (left) and 1 (right), $K=K_{M}$ (Michell

thin-ship approximation) and a simple ship bow form with

$B=12^{\circ}, \gamma=45^{\circ}$ and $F=0.5$.

12b. Real (top) and imaginary (bottom) parts of the functions $K_{ \pm} a_{k}^{t} /$ $\left(\mp \Theta_{ \pm}^{\prime}\right)^{1 / 2}$ for $k=2$ (left) and 3 (right), $K=K_{M}$ (Michel1 thin-ship approximation) and a simple ship bow form with $\beta=12^{\circ}, \gamma=45^{\circ}$ and $F=0.5$

13a. Real (top) and imaginary (bottom) parts of the functions $K_{ \pm} a_{k}^{\frac{t}{k}} /$ $\left(\mp \theta_{ \pm}^{\prime}\right)^{1 / 2}$ for $k=0$ (left) and 1 (right), $K=k_{0}$ (zeroth-order slender-ship approximation) and a simple ship bow form with $B=12^{\circ}, Y=45^{\circ}$ and $F=0.5$ 


\section{FIGURES (Continued)}

Page

13b. Real (top) and imaginary (bottom) parts of the functions $\mathrm{K}_{ \pm} \mathrm{a} \frac{ \pm}{\mathrm{k}} /$ $\left(\mp \Theta_{ \pm}^{\prime \prime}\right)^{1 / 2}$ for $k=2$ (left) and 3 (right), $K=K_{0}$ (zeroth-order slender-ship approximation) and a simple ship bow form with

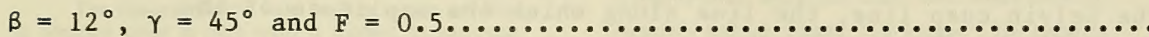

14. Real (top) and imaginary (bottom) parts of the functions $\mathrm{K}_{ \pm} \mathrm{a} \frac{ \pm}{4} /$ $\left(\mp \Theta_{ \pm}^{\prime \prime}\right)^{1 / 2}$ for $K_{M}($ left $)$ and $K_{0}(r i g h t)$ and a simple ship

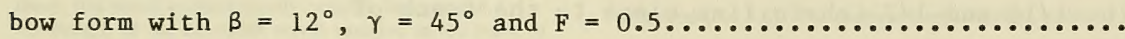

15. Amplitude of the transverse (-) and divergent (+) waves in the Kelvin wake for a simple ship bow form, with $\beta=12^{\circ}, \gamma=45^{\circ}$ and $F=0.5$, in the Michel1 thin-ship approximation (left) and the zeroth-order slender-ship approximation (right).

16. Steepness of the transverse $(-)$ and divergent $(+)$ waves in the Kelvin wake for a simple ship bow form, with $B=12^{\circ}, \gamma=45^{\circ}$ and $\mathrm{F}=0.5$, in the Michell thin-ship approximation (left) and the zeroth-order slender-ship approximation (right)

17. Upper bound for the steepness of the divergent waves in the Kelvin wake for a simple ship bow form, with $\beta=12^{\circ}, \gamma=45^{\circ}$ in the Michell thin-ship approximation (left) and the zeroth-order slender-ship

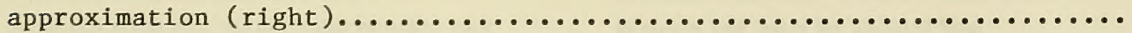

18. Steepness of the divergent waves in the Kelvin wake for a simple ship bow form, with $\beta=12^{\circ}, \gamma=0$ (top) and $45^{\circ}$ (bottom), and $\mathrm{F}=0.3$ (1eft) and 0.8 (right) in the zeroth-order slender-ship approximation 


\section{FIGURES (Continued)}

Page

19. Amplitude of the peak value of the steepness of the divergent waves in the Kelvin wake for a simple ship bow form, with $\beta=12^{\circ}$ and

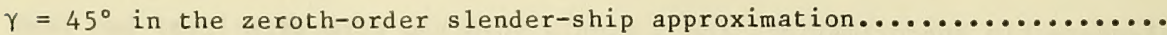

20. The Kelvin cusp line, the line along which the amplitude of the divergent waves exhibits a peak, and the three lines along which the steepness of the divergent waves is equal to $1 / 20,1 / 15$ and $1 / 7$ (chain line close to the track of the ship) for a simple ship bow form, with $\beta=12^{\circ}$ and $\gamma=45^{\circ}$, in the zeroth-order slender-ship

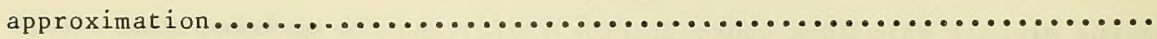

21. Lines along which the steepness of the divergent waves is equal to $1 / 20,1 / 15$ and $1 / 7$ for a simple ship bow form, with $\beta=12^{\circ}$ and $\gamma=45^{\circ}$, in the Michell thin-ship approximation (---) and the zeroth-order slender-ship approximation

22. Lincs along which the steepness of the divergent waves is equal to

$1 / 15$ for a simple ship bow form with $\beta=8^{\circ}, 12^{\circ}, 16^{\circ}$ and

$\gamma=45^{\circ}$ (top) and $\gamma=0,25^{\circ}, 45^{\circ}$ and $\beta=12^{\circ}$ (bottom)

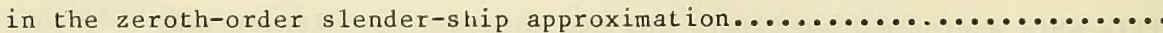




\title{
FAR-FIELD FEATURES OF THE KELVIN WAKF
}

\author{
A. Barnell and F. Noblesse
}

David W. Taylor Naval Ship Research and Ilevelopment Center

Bethesda, Maryland 20084

\begin{abstract}
The classical Kelvin wake trailing a ship advancing at constant speed in calm water is studied. In particular, asymptotic expressions for determining the far-field wake, at large distances behind the ship, are investigated numerically. This analysis, in which surface tension and nonlinearities are ignored, indicates that the divergent waves of a surface ship generally are infinitely steep at the track of the ship, even though their amplitude vanishes there; this result is theoretically possible because the wavelength of the divergent waves vanishes at the track of the ship. Inasmuch as infinitely-steep water waves cannot exist in reality, the linear no-surface-tension analysis presented in this study suggests that no divergent waves can exist within a certain region in the vicinity of the track of the ship, and that the Kelvin wake contains three distinct regions: (i) an inner region where only transverse waves can exist, (ii) an outer region where both transverse and divergent waves are present, and (iii) an intermediate region at the boundary between the inner and outer regions where short steep divergent waves, as well as transverse waves, can be found. Numerical results for a simple bow form show that the inner region is quite narrow, and that the wavelength of the divergent waves at the boundary of the inner region is of the order of $I$ to $20 \mathrm{~cm}$, depending on the speed of the ship. These results appear to be consistent with the narrow V-wakes observed in some SAR images of ship wakes. The analysis presented in this study may thus provide a partial explanation for these observations. However, the small wavelengths mentioned above indicate that the divergent waves in the vicinity of the track of the ship are likely to be significantly altered by surface tension, which should therefore be included in a more realistic analysis.
\end{abstract}

\section{INTRODUCTION}

It has been observed, e.g. $[1,2,3]$, that images of ship wakes taken by the SEASAT SAR (Synthetic

Aperture Radar) and other airborne SAR systems sometimes reveal long, narrow wakes extending back several miles behind ships. According to Swanson [3], "there appears a narrow, dark band along the centerline of the ship wake, widening slightly with distance away from the ship. Often accompanying these dark regions, at a slightly larger angle, are bright lines, which show up especially well on X-band SAR images". The apparent included angle of this narrow wake is much smaller that that corresponding in the Kelvin cusp line.

Several theoretical explanations of the features observed in SAR images of ship wakes have been proposed $[3,4,5,6]$. The proposed explanations include interactions between the cross currents created by a ship in its wake and surface gravity waves $[3,6]$ and the occurence of a sharp peak in the amplitude of the divergent waves in the Kelvin wake for a ship form having a large flare angle [5]. However, there currently appears to be no convincing electrodynamic or hydrodynamic explanation of the observations. It thus seems necessary to investigate in detail each one of the proposed possible explanations in order to determine whether one, or possibly a combination of several, of them does in fact provide a satisfactory theoretical interpretation of the SAR images of ship wakes.

The classical Kelvin wake trailing a ship advancing at constant speed in calm water is examined in this study. Viscosity, surface tension and nonlinearities are ignored in this preliminary analysis, and irrotational flow is assumed. The analysis and related numerical results for a simple ship bow form have led to several conclusions and recommendations for further studies which are presented at the end of the paper. Only two conclusions that may be related to some of the features observed in SAR images of ship wakes are discussed here.

It was previously found by Scragg [5] that, for a ship bow form with a large flare angle, the zeroth-order slender-ship approximation to the far-field wave-amplitude 
function given in Noblesse [7] predicts a sharp peak in the value of the amplitude of the divergent waves at an angle from the track of the ship equal to approximately half the bow entrance angle. This finding of Scragg has been verified in this study, as may be seen from figures 15,16 and 18. Furthermore, the magnitude of the waveamplitude peak has been found to increase very rapidly as the Froude number decreases below a certain threshold value. This peak thus appears to be a large-flare lowFroude-number feature. For the simple ship bow form considered in this study, the peak in the amplitude of the divergent waves in the Kelvin wake occurs along a line at $6^{\circ}$ from the track of the ship. It may be found from equation (30a) that the wavelength of the divergent waves along this line varies between $0.7 \mathrm{~m}$ and $4.5 \mathrm{~m}$ for a ship with speed varying between 10 knots and 25 knots, respectively.

The other conclusion of this study that may be related to the narow V-wakes observed in some SAR images of ship wakes is the result that the divergent waves of a surface ship are infinitely steep at the track of the ship, even though their amplitude vanishes there. This result is theoretically possible because the wavelength of the divergent waves vanishes at the track of the ship. A similar result was previously obtained by Sharma [8] who found that the Michell thin-ship approximation for a thin and deep strut-like ship form predicted infinite slopes for the divergent waves at the track of the ship. Inasmuch as infinitely-steep water waves cannot exist in reality, the foregoing result suggests that no divergent waves can exist within a certain region in the vicinity of the track of the ship, and that the Kelvin wake contains three distinct regions: (i) an inner region where only transverse waves can exist, (ii) an outer region where both transverse and divergent waves are present, and (iii) an intermediate region at the boundary between the inner and outer regions where short steep divergent waves, as well as transverse waves, can be found.

Numerical results for a simple bow form show that the "no-divergent-wave" inner region is quite narrow, as may be seen from figure 20 showing the Kelvin cusp line (angle $\simeq 19^{\circ} 28^{\prime}$ ), the line along which the amplitude of the divergent waves exhibits a peak (angle $\simeq 6^{\circ}$ ), and the three lines along which the steepness of the divergent waves is equal to $1 / 20,1 / 15$ and $1 / 7$ (chain line close to the track of the ship). The latter three lines, along which the divergent waves are steep, lie much closer to the track of the ship than the line along which the steepness of the divergent waves exhibits a peak. Figures 21 and 22 show considerable variations among the several "steep-divergentwaves" lines that are represented in these figures.

Nevertheless, these lines may be seen to correspond to values of $\mathrm{Y} /(-\mathrm{X})$ equal to about $10^{-2}$ to $2 \cdot 10^{-2}$. For ship speeds varying between 10 knots and 25 knots, the wavelength of the divergent waves corresponding to the foregoing values of $\mathrm{Y} /(-\mathrm{X})$ may be shown to vary between $0.7 \mathrm{~cm}$ and $4.2 \mathrm{~cm}$ for $\mathrm{Y} /(-\mathrm{X})=10^{-2}$, and $2.7 \mathrm{~cm}$ and $17 \mathrm{~cm}$ for $\mathrm{Y} /(-\mathrm{X})=2.10^{-2}$. These wavelengths are consistent with the wavelengths of the radar pulses used in SAR imaging, so that the foregoing results may provide a partial hydrodynamic explanation for the narrow $\mathrm{V}$-wakes observed in these images.

The tentative nature of this explanation must however be stressed. Indeed, the foregoing results are based on an analysis in which surface tension and nonlinearities have been neglected. Inasmuch as this linear no-surface-tension analysis predicts extremely short and steep waves in the vicinity of the track of the ship, it is evident that both surface tension and nonlinear effects are liable to be significant. In particular, the short wavelengths found along the steep-divergent-wave lines determined in this study, and the brief description of the effects of surface tension upon the Kelvin wake given in Sharma [8], Lamb [9, pp. 468-470] and Wehausen and Laitone [10, pp. 636-637] indicate that the system of divergent waves in the vicinity of the track of the ship is likely to be profoundly affected by surface tension. Effects of surface tension upon the Kelvin wake will be investigated in a sequel to the present study.

\section{APPROACH}

This study considers the steady potential flow due to a ship advancing with constant speed in calm water of infinite depth and lateral extent. The far-field Kelvin wake, which is of primary interest here, may be conveniently analyzed in terms of the nondimensional farfield coordinates $\vec{X}=\vec{X} g / U^{2}$, velocity potential $\phi=\Phi g / U^{3}$ and velocity vector $\nabla_{X} \phi=\nabla_{X} \Phi / U$, where $g$ is the gravitational acceleration and $\mathrm{U}$ is the speed of advance of the ship, $\vec{X}$ and $\Phi$ represent the dimensional coordinates and velocity potential, respectively, and $\nabla_{X}$ and $\nabla_{X}$ are the nondimensional and dimensional differential operators $\nabla_{\mathrm{x}}=(\partial / \partial \mathrm{x}, \partial / \partial \mathrm{y}, \partial / \partial \mathrm{z})$ and $\nabla_{\mathrm{X}}=(\partial / \partial \mathrm{X}, \partial / \partial \mathrm{Y}, \partial / \partial \mathrm{Z})$. The mean free surface is taken as the plane $z=0$, with 
the $\mathrm{z}$ axis pointing upwards, and the $\mathrm{x}$ axis is chosen in the ship centerplane and pointing towards the bow. The origin of the system of coordinates is placed within the ship. The Froude number is denoted by $F=U /(g L)^{1 / 2}$, where $\mathrm{L}$ is the length of the ship.

Equation (32) in [7] yields the following expression for the velocity potential associated with the Kelvin wake behind the ship

$\pi \phi(\vec{x})=\operatorname{Im} \int_{0}^{\infty}\left[E_{+}(\overrightarrow{t ; \vec{x}})+E_{-}(t ; \vec{x})\right] K(t) d t$,

where $E_{ \pm}(t ; \vec{x})$ is the exponential function

$E_{ \pm}(t ; \bar{x})=\exp \left[z\left(1+t^{2}\right)+i(x \pm y t)\left(1+t^{2}\right)^{1 / 2}\right]$,

and $K(t)$ is the far-field wave-amplitude function, which depends on the hull shape and the Froude number.

Assuming that differentiation under the integral sign is permitted in equation (1), we may obtain

$\pi\left\{\begin{array}{l}\phi_{x} \\ \phi_{y}\end{array}\right\}=\operatorname{Im~i} \int_{0}^{\infty}\left\{\begin{array}{l}E_{+}+E_{-} \\ E_{+}-E_{-}\end{array}\right\} K(t)\left\{\begin{array}{c}\left(1+t^{2}\right)^{1 / 2} \\ t\left(1+t^{2}\right)^{1 / 2}\end{array}\right\} d t$

The nondimensional elevation $\mathrm{e}=\mathrm{Eg} / \mathrm{U}^{2}$ of the

free surface at a sufficiently-large distance behind the ship, such that nonlinearities may be neglected, is given by

$e(x, y)=\partial \phi(x, y, 0) / \partial x$.

The slopes of the free surface in the directions parallel and perpendicular to the ship course then are

$\partial \mathrm{e}(\mathrm{x}, \mathrm{y}) / \partial \mathrm{x}=\partial^{2} \phi(\mathrm{x}, \mathrm{y}, 0) / \partial \mathrm{x}^{2}$

$\partial e(x, y) / \partial y=\partial^{2} \phi(x, y, 0) / \partial x \partial y$.

If differentiation under the integral sign in equation. (3a) is permitted, we have

$\left.\pi \mid \begin{array}{l}\phi_{x x} \\ \phi_{x y}\end{array}\right\}=-\operatorname{Im} \int_{0}^{\infty}\left|\begin{array}{l}E_{+}+E_{-} \\ E_{+}-E_{-}\end{array}\right| K(t)\left|\begin{array}{c}1+t^{2} \\ t\left(1+t^{2}\right)\end{array}\right| d t$.

The vertical velocity $\phi_{2}$ is given by

$\phi_{z}=-\phi_{x x}$.

In this study, we are mostly interested in the value of the several flow variables defined above at the mean free surface and behind the ship, so that we have $z=0$ and $x<0$. Expression (2) for the exponential function $E_{ \pm}$ then becomes

$E_{ \pm}=\exp \left[\operatorname{ix}(1 \mp \alpha t)\left(1+t^{2}\right)^{1 / 2}\right]$,

where $\alpha$ is defined as

$a=-\mathrm{y} / \mathrm{x}$.

For a ship with port- and starboard-symmetry, as is considered here, the Kelvin wake is symmetric about the ship track $y=0$. We may then restrict the analysis of the Kelvin wake to the domain $y \geqslant 0$ and $x<0$, and assume $\alpha \geqslant 0$.

Two difficulties associated with the foregoing approach for numerically determining the potential, its gradient, and the free-surface slopes in the Kelvin wake are readily apparent and should be noted here. A first numerical difficulty stems from the oscillations of the exponential function $\mathrm{E}_{ \pm}$given by equation (7), which are very rapid for large values of $|x|$. We have $x=\mathrm{Xg} / \mathrm{U}^{2}$ $=(\mathrm{X} / \mathrm{L}) / \mathrm{F}^{2}$. For a typical value of the Froude number equal to 0.2 , say, we thus have $x=25 \mathrm{X} / \mathrm{L}$; we then have $x=-250$ at 10 ship lengths behind the ship, and much larger values of $|x|$ must be considered at greater distances behind the ship or/and for smaller values of the Froude number. Even for the comparatively-moderate value of $x$ equal to -50 , figure 1 shows that the functions $\mathrm{E}_{+}$and $\mathrm{E}_{-}$oscillate quite rapidly. More precisely, figure 1 depicts the real parts of the functions $E_{+}(t ; x, \alpha)$ and $E_{-}(t ; x, \alpha)$ for $\mathrm{x}=-50 ; \alpha=.1, .2$, $1 / 2^{3 / 2}, .4$ and .5 ; and for $0 \leqslant t \leqslant 7$ and $0 \leqslant t \leqslant 3$ on the left and right sides, respectively. Figure 1 also indicates that the behavior of the function $\mathrm{E}_{+}(\mathrm{t} ; \mathrm{x}, \alpha)$ strongly depends on the value of $\alpha$.

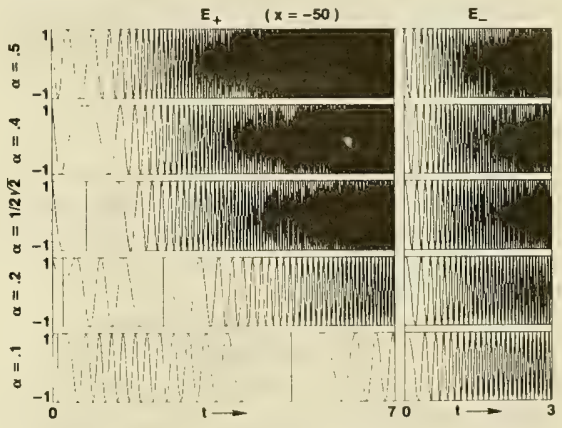

Fig. 1 - Real Parts of the Functions $\mathbf{E}_{+}(\mathrm{t} ; \mathrm{x}, \alpha)$ and E $(\mathrm{t} ; \mathrm{x}, \alpha)$ for $\mathrm{x}=-50$ and $\alpha=.1, .2,1 / 2^{3 / 2}, .4$ and .5

A second, more basic, difficulty is associated with the differentiation under the integral sign which was used for obtaining expressions $(3 a, b)$ and $(5 a, b)$ from expression (1). For a fully-submerged body, the far-field wave-amplitude function $\mathrm{K}(\mathrm{t})$ is exponentially-small as $\mathrm{t} \rightarrow$ $\infty$, so that the operation of differentiating under the integral sign in expression (1) can be continued indefinitely in principle. Differentiation under the integral sign likewise is justified if $z<0$. However, the operation must be justified in the limiting case $z=0$ for a surface ship. Clearly, the operation may not be justified in principle, or feasible in practice, if the far-field wave-amplitude 
function $K(t)$ does not vanish sufficiently rapidly as $t \rightarrow$ $\infty$. Precise information about the asymptotic behavior of the function $K(t)$ as $t \rightarrow \infty$ is required in this respect.

Figure 2 depicts the real and imaginary parts of the functions $\left(1+t^{2}\right) K(t)$ and $t\left(1+t^{2}\right) K(t)$ appearing in the integrands of the integrals ( $5 a)$ and $(5 b)$, respectively, for $0 \leqslant t \leqslant 16$ and for a simple ship-bow shape which is considered further on in this study. Differentiation under the integral sign in expression (3a) is clearly not justified in the case corresponding to figure 2 .

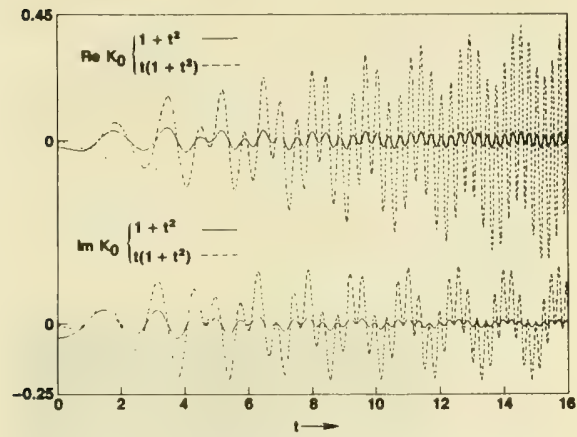

Fig. 2 - Real and Imaginary Parts of the Functions $\left(1+t^{2}\right) K(t)$ and $t\left(1+t^{2}\right) K(t)$ for a Simple Ship Bow Shape in the Zeroth-Order Slender-Ship Approximation

\section{ASYMPTOTIC EVALUATION OF THE KELVIN WAKE}

For large values of $|x|$ and $z=0$, analytical approximations to the integrals $(1),(3 a, b)$ and $(5 a, b)$ can be obtained by taking advantage of the rapid oscillations of the exponential functions $E_{+}$defined by equation (7). These functions may be expressed in the form $E=\exp [i x \theta(t ; \alpha)]$,

where $\theta(t ; \alpha)$ is defined as

$\theta(\mathrm{t} ; \alpha)=(1-\alpha \mathrm{t})\left(1+\mathrm{t}^{2}\right)^{1 / 2}$.

The functions $\mathrm{E}_{+}(\mathrm{t} ; \mathrm{X}, \alpha)$ and $\mathrm{E}_{-}(\mathrm{t} ; \mathrm{X}, \alpha)$, where $\alpha>0$, correspond to the function $\mathrm{E}(\mathrm{t} ; \mathrm{X}, \alpha)$ with $\alpha>0$ and $\alpha<0$, respectively. In the limiting case $\alpha=0$, we have $\mathrm{E}_{+}=$ $\exp \left[\operatorname{ix}\left(1+t^{2}\right)^{1 / 2}\right]=E_{-}$. The derivatives of the phasefunction $\theta(t ; x, \alpha)$ with respect to $t$ are given by

$\theta^{\prime}(\mathrm{t} ; \alpha)=-\left(\alpha-\mathrm{t}+2 \alpha \mathrm{t}^{2}\right) /\left(1+\mathrm{t}^{2}\right)^{1 / 2}$,

$\theta^{\prime \prime}(\mathrm{t} ; \alpha)=\left(1-3 \alpha \mathrm{t}-2 \alpha \mathrm{t}^{3}\right) /\left(1+\mathrm{t}^{2}\right)^{3 / 2}$,

$\theta^{\prime \prime \prime}(\mathrm{t} ; \alpha)=-3(\alpha+\mathrm{t}) /\left(1+\mathrm{t}^{2}\right)^{5 / 2}$.

The phase $\theta(t ; \alpha)$ and its first and second derivatives $\theta^{\prime}(t ; a)$ and $\theta^{\prime \prime}(t ; \alpha)$ are depicted in figures $3 a, b, c$, respectively.

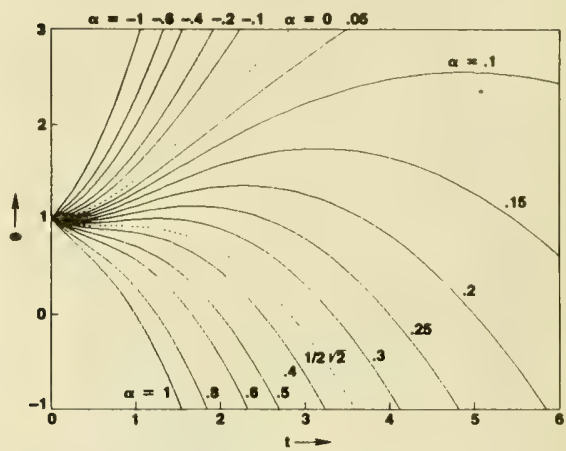

Fig. $3 a-$ The Phase Function $\theta(t ; \alpha)$ for $0 \leqslant t \leqslant 6$ and Several Values of $\alpha$

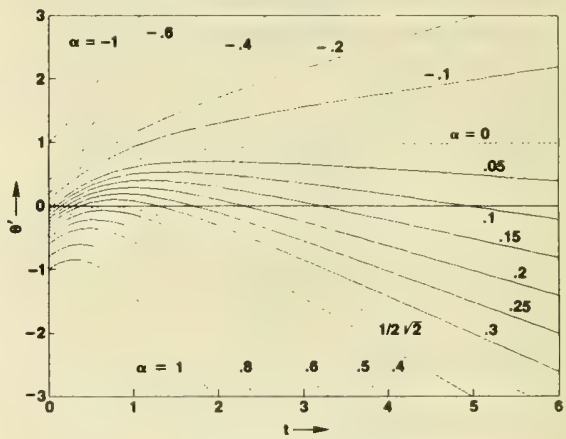

Fig. $3 b-$ The Function $\theta^{\prime}(t ; \alpha)$ for $0 \leqslant t \leqslant 6$ and Several Values of $a$

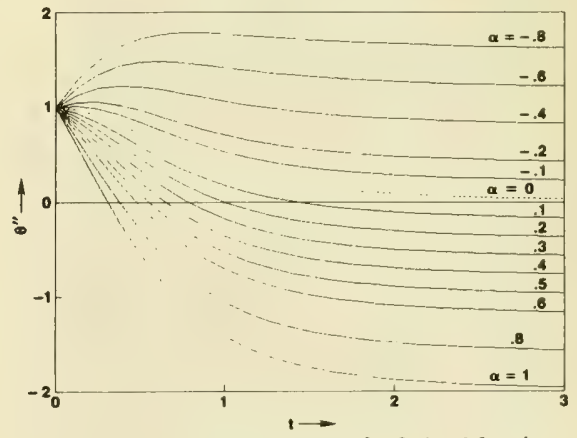

Fig. $3 c-$ The Function $\theta^{\prime \prime}(t ; \alpha)$ for $0 \leqslant t \leqslant 3$ and Several Values of $\alpha$ 
It may be seen from figure $3 b$ that the first derivative $\theta^{\prime}$ does not vanish except if $0 \leqslant \alpha \leqslant 1 / 2^{3 / 2}$, for which $\theta^{\prime}$ vanishe for 2 values of t. Equation (11a) shows that the 2 points where $\theta^{\prime}=0$, that is where the phase $\theta$ is stationary, are given by $t_{ \pm}(a)=\left[1 \pm\left(1-8 a^{2}\right)^{1 / 2}\right] / 4 a$.

We have $0 \leqslant t_{-} \leqslant t_{+} \leqslant \infty$, with $t_{-}=0$ and $t_{+}=\infty$ for $a=0$, and $t_{-}=1 / 2^{1 / 2}=t_{+}$for $\alpha=1 / 2^{3 / 2}$ The 2 points of stationary phase $t_{-}$and $t_{+}$are apparent on the left side of figure 1 for $\alpha=.1, .2$ and $1 / 2^{3 / 2}$. Figure $3 c$ shows that the second derivative $\theta^{\prime \prime}$ vanishes for one value of $t_{\text {, say }} t_{0}$, if $\alpha \geqslant 0$. Equation (11b) yields

$\mathrm{t}_{0}(a)=(\Gamma-1 / \Gamma) / 2^{1 / 2}$;

where $\Gamma$ is defined.as

$\Gamma=\left\{\left[1+\left(1+2 \alpha^{2}\right)^{1 / 2}\right] / 2^{1 / 2} a\right\}^{1 / 3}$

The value $t_{0}$ for which $\theta^{\prime \prime}=0$, and for which $\theta^{\prime}$ reaches its maximum as figure $3 \mathrm{~b}$ indicates, is a decreasing function of $\alpha$. We have $t_{0}=\infty$ for $\alpha=0, t_{0}=1 / 2^{1 / 2}$ for $\alpha=1 / 2^{3 / 2}$, and $t_{0}=0$ for $\alpha=\infty$. Furthermore, figure 4 , where the functions $t_{0}(\alpha), t_{-}(\alpha)$ and $t_{+}(\alpha)$ are depicted, shows that we have $0 \leqslant t_{-} \leqslant t_{0} \leqslant t_{+} \leqslant \infty$ for $0 \leqslant \alpha \leqslant 1 / 2^{3 / 2}$

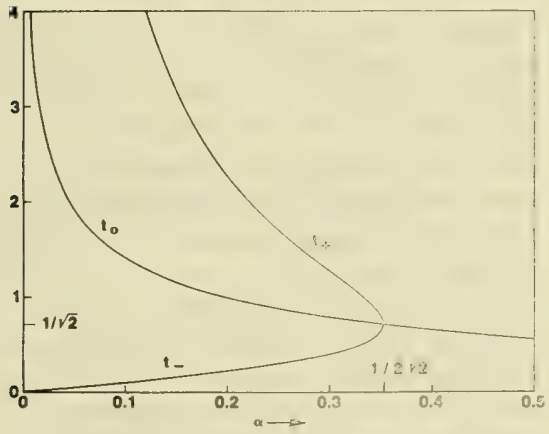

Fig. $4-$ The Functions $t_{0}(\alpha), t_{+}(\alpha)$ and $t_{-}(\alpha)$

In the vicinity of the points of stationary phase $t_{ \pm}$ the first derivative of the phase-function may be approximated by the two-term Taylor series $\theta^{\prime}(\mathrm{t} ; a) \simeq\left(\mathrm{t}-\mathrm{t}_{ \pm}\right) \theta_{ \pm}^{\prime \prime}+\left(\mathrm{t}-\mathrm{t}_{ \pm}\right)^{2} \theta_{ \pm}^{\prime \prime \prime} / 2$,

where the function $t_{ \pm}(a)$ is defined by equation (12), and $\theta_{ \pm}^{\prime \prime}$ and $\theta_{ \pm}^{\prime \prime \prime}$ represent the values of the functions $\theta^{\prime \prime}(t ; a)$ and $\theta^{\prime \prime \prime}(t ; \alpha)$ for $t=t_{ \pm}(a)$. By using equation (12) in equations (11b) and (11c) we may obtain $\theta_{ \pm}^{n}= \pm 2^{3 / 2} a\left(1-8 a^{2}\right)^{1 / 2} /\left[1+4 a^{2} \pm\left(1-8 a^{2}\right)^{1 / 2}\right]^{1 / 2}$, (15a)

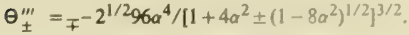

In the neighborhood of the point $t_{0}$, where $\theta^{\prime \prime}=0$, we have

$\theta^{\prime}(\mathrm{t} ; a) \simeq \theta_{0}^{\prime}+\left(\mathrm{t}-\mathrm{t}_{ \pm}\right)^{2} \theta_{0}^{\prime \prime \prime} / 2$,

where the function $t_{0}(\alpha)$ is defined by equation (13), and $\theta_{0}^{\prime}$ and $\theta_{0}^{\prime \prime \prime}$ represent the values of the functions $\theta^{\prime}(t ; \alpha)$ and $\theta^{\prime \prime \prime}(\mathrm{t} ; \alpha)$ defined by equations (11a) and (11c) for $\mathrm{t}=$ $t_{0}(\alpha)$. The functions $t_{0}(\alpha), t_{+}(\alpha)$ and $t_{-}(\alpha), \theta_{0}^{\prime}(a)$ and $\theta_{0}^{\prime \prime \prime}(\alpha), \theta_{+}^{\prime \prime}(\alpha)$ and $\theta^{\prime \prime}(\alpha), \theta_{+}^{\prime \prime \prime}(\alpha)$ and $\theta_{-}^{\prime \prime \prime}(\alpha)$ are depicted in figure 5. The signs of the functions $\theta_{0}^{\prime}, \theta_{0}^{\prime \prime \prime}, \theta_{ \pm}^{\prime \prime}$ and $\Theta_{ \pm}^{\prime \prime \prime}$ are readily apparent from this figure. The Taylorseries approximation (14) and (16) are useful for devising an efficient numerical method for evaluating the integrals (1), $(3 a, b)$ and $(5 a, b)$, as will be examined in detail elsewhere.

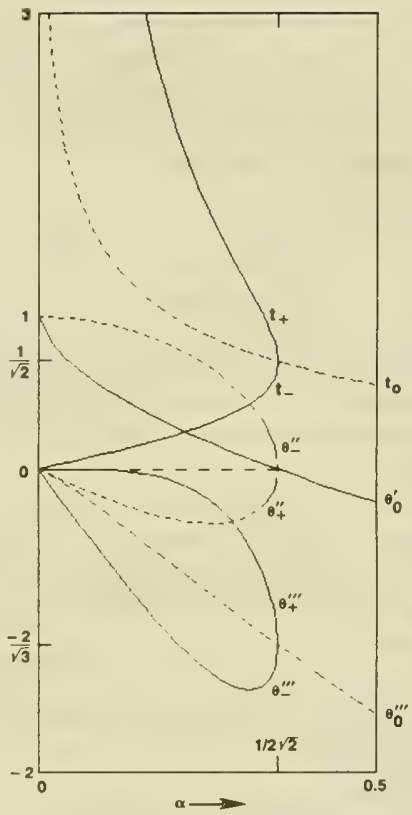

Fig. $5-$ The Functions $t_{0}(\alpha), t_{ \pm}(\alpha), \Theta_{0}^{\prime}(\alpha), \Theta_{0}^{\prime \prime \prime}(\alpha)$, $\Theta_{ \pm}^{\prime \prime}(\alpha)$ and $\Theta_{ \pm}^{\prime \prime \prime}(\alpha)$ for $0 \leqslant \alpha \leqslant 0.5$

Asymptotic approximations valid for $z=0$ and $x$ $\rightarrow-\infty$ will now be obtained for the integrals $(1),(3 a, b)$ and $(5 a, b)$. These five integrals may be expressed in the form

$\pi \phi_{\mathrm{k}}(\mathrm{x}, \alpha)=\operatorname{Im}\left(\mathrm{c}_{\mathrm{k}}^{+} \psi_{\mathrm{k}}^{+}+\mathrm{c}_{\mathrm{k}}^{-} \psi_{\mathrm{k}}^{-}\right)$.

where $0 \leqslant k \leqslant 4, c_{k}^{ \pm}$are constants, and $\psi_{k}^{ \pm}$are the 
integrals defined as

$\psi_{k}^{ \pm}(x, a)=\int_{0}^{\infty} \exp \left[i-\theta_{\perp}(i ; f)\right] K(t) a_{k}(t) d t ;$

in this expression, tive phase $\theta_{ \pm}(t ; a)$ is defined by $\theta_{ \pm}(t ; \alpha)=(1 \mp a t)\left(1+t^{2}\right)^{1 / 2}$ with $\alpha \geqslant 0$;

finally, the constants $c_{k}^{ \pm}$in equation (17) and the functions $a_{k}(t ;$; in ertuation (18) are defined in the following table:

$\begin{array}{ccccc}k & \phi_{k} & a_{k} & c_{k}^{+} & c_{k}^{-} \\ 0 & \phi_{x}^{-} & 1 & 1 & 1 \\ 1 & \phi_{x} & \left(1+t^{2}\right)^{1 / 2} & i & i \\ 2 & \phi_{y} & t\left(1+t^{2}\right)^{1 / 2} & i & -i \\ 3 & \phi_{x x} & 1+t^{2} & -1 & -1 \\ 4 & \phi_{x y} & t\left(1+t^{2}\right) & -1 & 1\end{array}$

In the particular case $\alpha=0$, that is on the track of the ship, equation (18) yields $\psi_{\mathrm{k}}^{+}=\psi_{\mathrm{k}}=\psi_{\mathrm{k}}^{-}$, with $\psi_{k}(x, 0)=\int_{0}^{\infty} \exp \left[\operatorname{ix}\left(1+t^{2}\right)^{1 / 2}\right] K(t) a_{k}(t) d t$.

The major contribution to this integral in the limit $x \rightarrow$ $-\infty$ stems from the point of stationary phase at the origin $t=0$. Specifically, we may obtain

$(\pi / 2)^{1 / 2}(-x)^{1 / 2} \phi_{k}(x, 0) \sim$

Im $c_{k} K_{0} \exp [i(x-\pi / 4)]$ as $x \rightarrow-\infty$,

with $\mathrm{c}_{0}=1, \mathrm{c}_{1}=\mathrm{i}, \mathrm{c}_{3}=-1$,

and $c_{2}=0=c_{4}$; we thus have

$\phi_{y}=0=\phi_{x y}$ for $\alpha=0$,

in accordance with the symmetry of the wave pattern

about the axis $\alpha=0$. In equation (21), $\mathrm{K}_{0}$ represents the value of the function $K(t)$ at the origin $t=0$, that is we have $\mathrm{K}_{0}=\mathbf{K}(0)$.

For $0<\alpha<1 / 2^{3 / 2}$, the phase $\theta_{+}(\mathrm{t} ; \alpha)$ is stationary, that is $\theta_{+}^{\prime}=0$, at the two distinct points $t_{-}(\alpha)$ and $t_{+}(\alpha)$ defined by equation (12), whereas $\theta_{-}^{\prime}(t ; \alpha)>0$ for $t \geqslant 0$, as may be seen from figure $3 \mathrm{~b}$. Equations (17) and (18) then yield

$\pi \phi_{k}(x, a) \sim \operatorname{Im~} c_{k}^{+} \psi_{k}^{+}$as $x \rightarrow-\infty$ with $0<a<1 / 2^{3 / 2}$.

The contribution of the two points of stationary phase $t_{ \pm}$ can be evaluated by using the method of stationary phase, with the result

$(\pi / 2)^{1 / 2}(-x)^{1 / 2} \phi_{k}(x, \alpha) \sim \operatorname{Im} c_{k}\left(A_{k}^{-} E_{-}+A_{k}^{+} E_{+}\right)$

as $\mathrm{x} \rightarrow-\infty$, with $0<\alpha<1 / 2^{3 / 2}$ and

$c_{0}=1, c_{1}=i=c_{2}, c_{3}=-1=c_{4}$;

furthermore, $\mathbf{A}_{k}^{ \pm}$and $\mathrm{E}_{ \pm}$are the amplitude and exponential functions defined as

$\mathbf{A}_{\mathbf{k}}^{ \pm}=\mathrm{K}_{ \pm} \mathrm{a}_{\mathrm{k}}^{ \pm} /\left(\mp \theta_{ \pm}^{\prime \prime}\right)^{1 / 2}$,

$\mathrm{E}_{ \pm}=\exp \left[\mathrm{i}\left(\mathrm{x} \Theta_{ \pm} \pm \pi / 4\right)\right]$,

where $K_{ \pm}, a_{k}^{ \pm}, \theta_{ \pm}$and $\theta_{ \pm}^{\prime \prime}$ are defined as

$\mathbf{K}_{ \pm}=\mathbf{K}\left(\mathbf{t}_{ \pm}\right), \mathbf{a}_{\mathbf{k}}^{ \pm}=\mathbf{a}_{\mathbf{k}}\left(\mathrm{t}_{ \pm}\right)$, $\theta_{ \pm}=\theta\left(t_{ \pm} ; a\right), \theta_{ \pm}^{\prime \prime}=\theta^{\prime \prime}\left(t_{ \pm} ; \alpha\right)$,

(2Sc,d)

and $t_{ \pm}$is the function of a given by equation (12), that is we have

$t_{ \pm}=\left[1 \pm\left(1-8 \alpha^{2}\right)^{1 / 2}\right] / 4 a$.

We may then obtain

$1+\mathrm{t}_{ \pm}^{2}=\left[1+4 \alpha^{2} \pm\left(1-8 \alpha^{2}\right)^{1 / 2}\right] / 8 \alpha^{2}$.

The expressions for the terms $\mathrm{a}_{\mathbf{k}}^{ \pm}$may readily be obtained from equations $(20 \mathrm{a}-\mathrm{e}),(25 \mathrm{~b})$ and $(26 \mathrm{a}, \mathrm{b})$. Equations $(25 \mathrm{c})$, (19) and $(26 \mathrm{a}, \mathrm{b})$ yield

$\theta_{ \pm}=\left[3 \mp\left(1-8 a^{2}\right)^{1 / 2}\right]\left[1+4 a^{2} \pm\left(1-8 a^{2}\right)^{1 / 2}\right]^{1 / 2} / 2^{1 / 2} 8 \alpha$. (26c)

Finally, $\theta_{ \pm}^{\prime \prime}$ is given by equation (15a), that is we have $\mp \theta_{ \pm}^{\prime \prime}=2^{3 / 2} \alpha\left(1-8 \alpha^{2}\right)^{1 / 2} /\left[1+4 a^{2} \pm\left(1-8 \alpha^{2}\right)^{1 / 2}\right]^{1 / 2}$.

It may be shown from equations $(26 a, c, d)$ and

verified from figures $4,3 a, 3 c$ and 5 that we have $t_{-}=0$ and $\theta_{-}=1=\theta_{-}^{\prime \prime}$ in the limit $\alpha=0$. Furthermore, equations (25b) and (20a-e) show that we have $a_{k}^{-}=1$ for $k=0,1$ and 3 , and $a_{k}^{-}=0$ for $k=2$ and 4 in the limit $a=0$. The asymptotic approximation (23) for $\phi_{k}(\mathrm{x}, \alpha)$ therefore becomes identical to the asymptotic approximation given by equations (21), $(21 \mathrm{a}, \mathrm{b}, \mathrm{c})$ and $(22 \mathrm{a}, \mathrm{b})$ for $\phi_{\mathrm{k}}(\mathrm{x}, 0)$ in the limit $\alpha=0$, if the contribution of the second point of stationary phase $t_{+}=\infty$ is null, that is if $\mathbf{A}_{\mathrm{k}}^{+}=0$ for $\alpha=0$ and $\mathrm{t}_{+}=\infty$. In other words, the asymptotic approximation (23) for $\phi_{\mathrm{k}}(\mathrm{x}, a)$ is uniformly valid in the vicinity of the track of the ship $\alpha=0$ if $\mathrm{A}_{\mathrm{k}}^{+}=$ 0 for $\alpha=0$. Equations (26a) and (26d) yield $t_{+} \sim 1 / 2 \alpha$ and $-\theta_{+}^{\prime \prime} \sim 2 \alpha$ as $\alpha \rightarrow 0$. We then have $-\theta_{+}^{\prime \prime} \sim 1 / t_{+}$ as $\alpha \rightarrow 0$, and the condition for the asymptotic approximation for $\phi_{k}(x, a)$ to be uniformly valid in the limit $a=0$ takes the form

$\mathrm{t}^{1 / 2} \mathrm{~K}(\mathrm{t}) \mathrm{a}_{\mathrm{k}}(\mathrm{t}) \rightarrow 0$ as $\mathrm{t} \rightarrow \infty$.

In the limit $\alpha=1 / 2^{3 / 2}$, we have $t_{-}=1 / 2^{1 / 2}=$

$\mathrm{t}_{+}$and $\Theta_{ \pm}^{\prime \prime}=0$, as may be verified from equations $(26 a, d)$ and figure 5. Equation (24a) then shows that have $\left|A_{k}^{ \pm}\right| \rightarrow \infty$ as $\alpha \rightarrow 1 / 2^{3 / 2}$; and the asymptotic approximation (23) is not valid in the vicinity of the boundary of the Kelvin wake. A complementary asymptotic approximation, expressed in terms of Airy functions, valid at and near the Kelvin cusp line is given in Ursell [11] for the particular case of a pressure point at the free surface. However, we are mostly interested in the sector $0 \leqslant \alpha<1 / 2^{3 / 2}$, that is inside the Kelvin wake, in the present study.

The far-field asymptotic approximation (23) shows that the wave pattern at any point $(\mathrm{x}, \alpha)$, with $\mathrm{x} \ll-1$ and $0 \leqslant a<1 / 2^{3 / 2}$, consists in two elementary plane progressive waves. Specifically, equations (4), (20b), (23) 
and (24b) show that the free-surface elevation within the

Kelvin wake at a sufficiently-large distance behind the ship is given by

$(\pi / 2)^{1 / 2}(-x)^{1 / 2} e(x, \sigma) \sim \operatorname{Re}\left[A_{1} \exp (i \varphi)+A_{1}^{+} \exp \left(i \varphi_{+}\right)\right]$, where the phases $\varphi_{ \pm}$of the two waves are given by $\varphi_{ \pm}=x \theta_{ \pm} \pm \pi / 4$. Curves along which the phases $\varphi_{+}$or $\varphi_{\text {- }}$ are constant then are defined by the equation $x \Theta_{ \pm} \pm \pi / 4=$ constant. This relation and equation (8) then yield the following parametric equations for the curves along which the phase is equal to $-2 \mathrm{n} \pi$ :

$-\mathrm{x}_{ \pm}=(2 \mathrm{n} \pi \pm \pi / 4) / \Theta_{ \pm}$,

$y_{ \pm}=(2 n \pi \pm \pi / 4) a / \theta_{ \pm}$,

where $0 \leqslant \alpha \leqslant 1 / 2^{3 / 2}$. The ten constant-phase curves corresponding to $1 \leqslant n \leqslant 10$ are depicted in figure 6 . The "transverse" and "divergent" waves in this classical representation of the Kelvin wake correspond to the waves $A_{1}^{-} \exp \left(i \varphi_{-}\right)$and $A_{1}^{+} \exp \left(i \varphi_{+}\right)$, respectively, in equation (28).

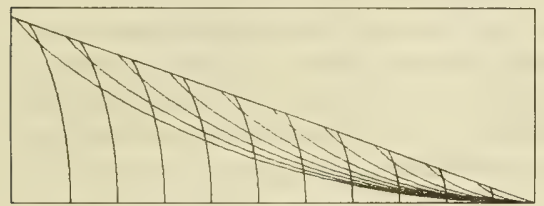

Fig. 6 - The Classical Kelvin Ship Wave Pattern

The wavenumber corresponding to the wave with phase $\varphi_{ \pm}$is given by $\nabla \varphi_{ \pm}$. The corresponding wavelength, say $\lambda_{ \pm}$, and direction of propagation with respect to the track of the ship, say $\beta_{ \pm}$, then are given by $\lambda_{ \pm}=2 \pi /\left|\nabla \varphi_{ \pm}\right|$and $\beta_{ \pm}=\tan ^{-1}\left(\varphi_{y}^{ \pm} / \varphi_{x}^{ \pm}\right)$, where $\varphi_{x}^{ \pm}$and $\varphi_{y}^{ \pm}$represent the $x$ - and y-derivatives of $\varphi_{ \pm}$and $\left|\nabla \varphi_{ \pm}\right|$ $=\left[\left(\varphi_{x}^{ \pm}\right)^{2}+\left(\varphi_{y}^{ \pm}\right)^{2}\right]^{1 / 2}$. The relation $\varphi_{ \pm}=x \Theta_{ \pm} \pm \pi / 4$ and equation (26c) then yield

$\lambda_{ \pm}=2^{1 / 2} 16 \pi \alpha^{2} /\left[3 \mp\left(1-8 \alpha^{2}\right)^{1 / 2}\right]\left[1-4 \alpha^{2} \pm\left(1-8 \alpha^{2}\right)^{1 / 2}\right]^{1 / 2}$,

$\beta_{ \pm}=\sin ^{-1}\left\{\left[1-4 \alpha^{2} \pm\left(1-8 \alpha^{2}\right)^{1 / 2}\right] /\left[1+4 a^{2} \pm\left(1-8 \alpha^{2}\right)^{1 / 2}\right]\right\}^{1 / 2}$.

Equations $(30 \mathrm{a}, \mathrm{b})$ show that we have $\lambda_{-}=2 \pi, \beta_{-}=0$

and $\lambda_{+}=0, \beta_{+}=\pi / 2$ in the limit $\alpha=0$, and $\lambda_{-}=$

$4 \pi / 3=h_{+}, \beta_{-}=\sin ^{-1}\left(1 / 3^{1 / 2}\right)=\beta_{+}$for $a=1 / 2^{3 / 2}$.

More precisely, we have

$2 \pi \geqslant \lambda_{-} \geqslant 4 \pi / 3 \geqslant \lambda_{+} \geqslant 0$ and

$0 \leqslant \beta_{-} \leqslant \sin ^{-1}\left(1 / 3^{1 / 2}\right) \leqslant \beta_{+} \leqslant \pi / 2$,

as may be seen from figure 7 where the functions

$\lambda_{ \pm}(\alpha) / 2 \pi$ and $2 \beta_{ \pm}(\alpha) / \pi$ are depicted.

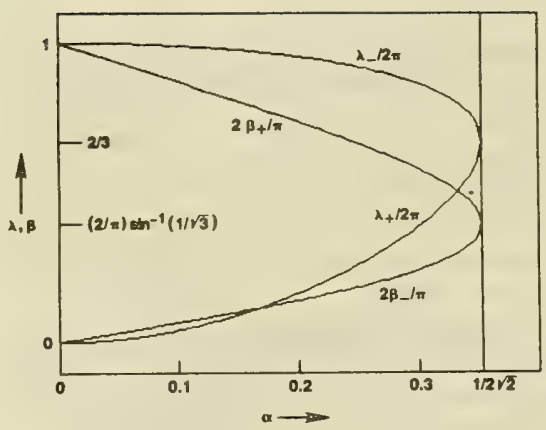

Fig. 7 The Wavelengths $\lambda_{ \pm}(\alpha)$ and Propagation Angles $\beta_{ \pm}(\alpha)$ of the Transverse and Divergent Waves in the Kelvin Wake

Equation (28) shows that the amplitudes of the transverse and divergent waves in the Kelvin wake are asymptotically given by $(2 / \pi)^{1 / 2}\left|A_{1}^{ \pm}\right| /(-x)^{1 / 2}$ as $x \rightarrow-\infty$. The steepnesses, say $s_{ \pm}$, of these waves then are given by $s_{ \pm}=(2 / \pi)^{1 / 2}\left|A_{1}^{ \pm}\right| /(-x)^{1 / 2} \lambda_{ \pm} \cdot$ Equations $(24 a),(25 a, b)$ and $(20 \mathrm{~b})$ then yield $(-x)^{1 / 2} s_{ \pm} \sim \sigma_{ \pm}(a)\left|K\left(t_{ \pm}\right)\right|$as $x \rightarrow-\infty$, where $\sigma_{ \pm}(\alpha)$ is defined as $(2 / \pi)^{1 / 2}\left(1+t_{ \pm}^{2}\right)^{1 / 2} /\left(\mp \theta_{ \pm}^{\prime \prime}\right)^{1 / 2} \lambda_{ \pm}$.

Equations $(26 \mathrm{~b}, \mathrm{~d})$ and $(30 \mathrm{a})$ then yield

$o_{ \pm}=\left[3 \mp\left(1-8 \alpha^{2}\right)^{1 / 2}\right]\left[1-4 \alpha^{2} \pm\left(1-8 \alpha^{2}\right)^{1 / 2}\right]^{1 / 2}$

$\left[1+4 \alpha^{2} \pm\left(1-8 \alpha^{2}\right)^{1 / 2}\right]^{3 / 4} /$

$64 \pi\left(2^{1 / 2} \pi\right)^{1 / 2} \alpha^{7 / 2}\left(1-8 \alpha^{2}\right)^{1 / 4}$.

Equations (12) and (33) yield $t_{-}=0$ and $\sigma_{-}=$ $1 / \pi(2 \pi)^{1 / 2}$ in the limit $\alpha=0$, for which we have $\lambda_{-}=2 \pi$ and $\beta_{-}=0$ as was noted previously. The steepness of the transverse wave at a point $(x, 0)$ on the track of the ship then is given by

s_ $(x, 0) \sim|K(0)| / \pi(2 \pi)^{1 / 2}(-x)^{1 / 2}$ as $x \rightarrow-\infty$.

Equations (12) and (33) also yield $t_{+} \sim 1 / 2 \alpha$ and $\sigma_{+} \sim 1 / 16 \pi^{3 / 2} \alpha^{7 / 2}$ in the limit $\alpha \rightarrow 0$, for which we have $\lambda_{+} \rightarrow 0$ and $\beta_{+} \rightarrow \pi / 2$. We thus have $\sigma_{+} \sim t_{+}^{7 / 2} / \pi(2 \pi)^{1 / 2}$ as $a \rightarrow 0$, and equation (33) shows that the steepness of the divergent wave at a point $(x, a)$ in the vicinity of the track of the ship is given by

$\mathrm{s}_{+}(\mathrm{x}, \alpha) \sim \mathrm{t}_{+}^{7 / 2}\left|\mathrm{~K}\left(\mathrm{t}_{+}\right)\right| / \pi(2 \pi)^{1 / 2}(-\mathrm{x})^{1 / 2}$

as $\alpha \rightarrow 0$, with $t_{+} \sim 1 / 2 \alpha$.

The steepness $s_{+}(x, \alpha)$ then becomes unbounded as $\alpha \rightarrow 0$ if

$t^{7 / 2}|K(t)| \rightarrow \infty$ as $t \rightarrow \infty$. 
Equations (4), (20b) and (27) show that the asymptotic expansion (23) for the free-surface elevation is valid in the vicinity of the track of the ship if

$\mathrm{t}^{3 / 2} \mathrm{~K}(\mathrm{t}) \rightarrow 0$ as $\mathrm{t} \rightarrow \infty$.

Let us assume that we have

$|\mathrm{K}(\mathrm{t})| \sim 1 / \mathrm{t}^{\mu}$ as $\mathrm{t} \rightarrow \infty$.

Both conditions (37) and (36) are then satisfied if $3 / 2<\mu<7 / 2$.

Condition (37) means that the amplitudes of the divergent waves in the Kelvin wake vanish as $\alpha \rightarrow 0$, that is as the track of the ship is approached, whereas condition (36) means that the waves become infinitely steep; this is theoretically possible because the wavelengths of the divergent waves vanish as $\alpha \rightarrow 0$, as is indicated in equation (31a) and figure 7. However, infinitely-steep water waves cannot exist in reality; indeed, there exists a theoretical upper bound for the steepness of water waves in deep water which is approximately equal to $1 / 7$. Condition (39) therefore suggests that no divergent waves can exist within a certain domain in the vicinity of the track of the ship, and that the Kelvin wake contains three distinct regions: (i) an inner region adjacent to the track of the ship where only transverse waves can exist as was just noted, (ii) an outer region where both transverse and divergent waves are present, and (iii) a region at the boundary between the inner and outer regions where short steep divergent waves, as well as transverse waves, can be found. It must be kept in mind, however, that these conclusions regarding the Kelvin wake are based on condition (39), which was obtained on the basis of an analysis in which surface-tension and nonlinearities are ignored. Inasmuch as this linear no-surface-tension analysis predicts short steep waves, both surface-tension and nonlinear effects are liable to be significant, and these effects should therefore be included in a more realistic analysis. In particular, it is evident from Lamb [9, pp. 468-470] and Wehausen and Laitone [10, pp. 636-637] that the system of divergent waves in the immediate vicinity of the track of the ship may be profoundly affected by surface tension.

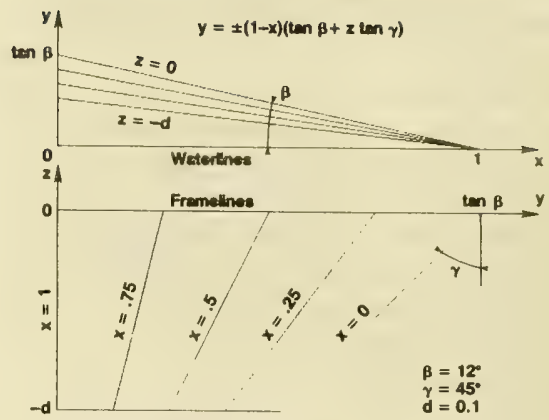

Fig. 8 - Waterlines and Framelines of the Simple Ship Bow Form Considered for Numerical Applications

\section{A SIMPLE TEST CASE:}

THE FAR-FIELD WAVE-AMPLITUDE FUNCTION

The foregoing theoretical results are investigated numerically for the simple semi-infinite ship form studied previously by Scragg [5]. This ship form consists of a bow region, with length $\mathrm{L}$, followed by a parallel body, with invariant framelines, extending to infinity downstream. All framelines, both in the bow region and downstream from it, are trapezoidal in shape with constant draft D. The waterlines are rectilinear. More precisely, the hull form is defined by the equations

$\mathrm{y}= \pm(\tan \beta+\mathrm{z} \tan \gamma)(1-\mathrm{x})$

for $0 \leqslant x \leqslant 1$ and $0 \geqslant z \geqslant-d$,

$y= \pm(\tan \beta+z \tan \gamma)$ for $x \leqslant 0$ and $0 \geqslant z \geqslant-d$,

where $x, y, z$ and $d$ are nondimensional in terms of the length of the bow region, that is we have $\vec{x}=\vec{X} / L$ and $\mathrm{d}=\mathrm{D} / \mathrm{L}$. Equations $(40 \mathrm{a}, \mathrm{b})$ require that the condition $\tan \beta \geqslant d \tan \gamma$

be satisfied. Equation (40a) shows that the entrance angle at the bow ( $x=1, z=0$ ) is equal to $2 \beta$, and it may be seen from equations $(40 a, b)$ that $y$ represents the flare angle for $\mathrm{x} \leqslant 0$. The four waterlines corresponding to $z=0,-d / 3,-2 d / 3,-d$ and the five framelines corresponding to $x=1,0.75,0.5,0.25,0$ are depicted in figure 8 for $d=0.1, \beta=12^{\circ}$ and $\gamma=45^{\circ}$. The notation 
$\beta_{0}=\tan \beta, \gamma_{0}=\tan \gamma$

will be used for shortness hereafter.

The far-field wave-amplitude function $\mathbf{K}(\mathrm{t})$ for the foregoing semi-infinite hull has been evaluated for two simple approximations defined explicitly in terms of the hull shape and the Froude number, namely the Michell thin-ship approximation and the zeroth-order slender-ship approximation [7], for which the function $K(t)$ is denoted $K_{M}(t)$ and $K_{0}(t)$, respectively. The Michell thin-ship approximation is given by the product of two single integrals, as follows:

$\mathrm{K}_{\mathrm{M}}=2 v^{4} \int_{0}^{1} \exp \left[-\mathrm{i}^{2}\left(1+\mathrm{t}^{2}\right)^{1 / 2} \mathrm{x}\right] \mathrm{dx}$
$\int_{-\mathrm{d}}^{0} \exp \left[v^{2}\left(1+\mathrm{t}^{2}\right) \mathrm{z}\right]\left(\beta_{0}+\gamma_{0} \mathrm{z}\right) \mathrm{dz}$.

These integrals can be evaluated analytically, with the result

$K_{M}(t)=4\left[\beta_{0}-\left(\beta_{0}-\gamma_{0} d\right) e-F^{2} \gamma_{0}(1-e) /\left(1+t^{2}\right)\right]$

$\sin \left[v^{2}\left(1+t^{2}\right)^{1 / 2} / 2\right] \exp \left[-i v^{2}\left(1+t^{2}\right)^{1 / 2} / 2\right] /\left(1+t^{2}\right)^{3 / 2}$,

where the term $e$ is defined as

$\mathrm{e}=\exp \left[-v^{2} \mathrm{~d}\left(1+\mathrm{t}^{2}\right)\right]$.

Equations (43) and (44) yield

$\left|\mathrm{K}_{\mathrm{M}}(\mathrm{t})\right| \sim 4 \beta_{0}\left|\sin \left(v^{2} \mathrm{t} / 2\right)\right| / \mathrm{t}^{3}$ as $\mathrm{t} \rightarrow \infty$.

The zeroth-order slender-ship approximation may be expressed in the form

$\mathrm{K}_{0}(\mathrm{t})=\mathrm{K}_{0}^{+}(\mathrm{t})+\mathrm{K}_{0}^{-}(\mathrm{t})$,

where $K_{0}^{ \pm}(t)$ is given by the sum of a double integral over the hull surface and a single integral along the top waterline, as follows:

$$
\begin{aligned}
\mathrm{K}_{0}^{ \pm}= & v^{4} \int_{0}^{1} \mathrm{dx} \int_{-\mathrm{d}}^{0} \mathrm{dz} \exp \left[v^{2}\left(1+\mathrm{t}^{2}\right) \mathrm{z}\right] \\
& \exp \left[-\mathrm{i} v^{2}\left(1+\mathrm{t}^{2}\right)^{1 / 2}(\mathrm{x} \pm \mathrm{yt})\right]\left(\beta_{0}+\gamma_{0} \mathrm{z}\right) \\
& -v^{2} \int_{0}^{1} \mathrm{dx} \exp \left[-\mathrm{i} \nu^{2}\left(1+\mathrm{t}^{2}\right)^{1 / 2}(\mathrm{x} \pm \mathrm{yt})\right] \\
& \beta_{0}^{3} /\left[1+\beta_{0}^{2}+\gamma_{0}^{2}(1-\mathrm{x})^{2}\right] .
\end{aligned}
$$

The integration in these integrals is carried over the positive half of the hull surface. Equations (40a) and $(42 \mathrm{a}, \mathrm{b})$ then yield $\mathrm{y}=\left(\beta_{0}+\gamma_{0} \mathrm{z}\right)(1-\mathrm{x})$. We then have $x \pm y t=1-\left(1 \pm \beta_{0} t\right)(1-x) \pm y_{0} t(1-x) z$

By using this relation into equation (47) we may then obtain

$$
\begin{gathered}
\exp \left[i v^{2}\left(1+t^{2}\right)^{1 / 2} \mid K_{0}^{ \pm}(t)=v^{2} \int_{0}^{1} \exp \left[i v^{2}\left(1+t^{2}\right)^{1 / 2}\left(1 \mp \beta_{0} t\right) x\right]\right. \\
\cdot\left[\beta_{0} I_{0}^{ \pm}-\gamma_{0} I_{1}^{ \pm}-\beta_{0}^{3} /\left(1+\beta_{0}^{2}+\gamma_{0}^{2} x^{2}\right)\right] d x
\end{gathered}
$$

where $\mathrm{I}_{\mathrm{n}}^{ \pm}$, with $\mathrm{n}=0$ and 1 , are the integrals defined as $\left.(-1)^{\mathrm{n}}\right]_{\mathrm{n}}^{ \pm}=v^{2} \int_{-\mathrm{d}}^{0} \exp \left[v^{2}\left(1+\mathrm{t}^{2}\right)\left(1 \mp \mathrm{i} \gamma_{0} \mathrm{ux}\right) \mathrm{z}\right] \mathrm{z}^{\mathrm{n}} \mathrm{dz}$, with $u$ defined as

$u=t /\left(1+t^{2}\right)^{1 / 2}$.

The integrals $I_{n}^{ \pm}$may be evaluated analytically with the result

$\mathrm{I}_{0}^{ \pm}=\left(1-\mathrm{ee}_{ \pm}\right) /\left(1+\mathrm{t}^{2}\right)\left(1 \mp i \gamma_{0} \mathrm{ux}\right)$,

$\mathrm{I}_{1}^{ \pm}=\left[\mathrm{F}^{2}\left(1-\mathrm{ee} e_{ \pm}\right) /\left(1+\mathrm{t}^{2}\right)\left(1 \mp \mathrm{i} \gamma_{0} \mathrm{ux}\right)-\mathrm{dee}_{ \pm}\right]$ $/\left(1+\mathrm{t}^{2}\right)\left(1 \mp \mathrm{ir} \mathrm{y}_{0} \mathrm{ux}\right)$,

where $e$ is the exponential function given by equation (44) and $e_{ \pm}$is the exponential function defined as

$e_{ \pm}=\exp \left[ \pm i v^{2} d \gamma_{0} t\left(1+t^{2}\right)^{1 / 2} x\right]$.

We then have

$\beta_{0} \mathrm{I}_{0}^{ \pm}-\gamma_{0} \mathrm{I}_{\mathrm{I}}^{ \pm}-\beta_{0}^{3} /\left(1+\beta_{0}^{2}+\gamma_{0}^{2} \mathrm{x}^{2}\right)=$

$\mathrm{A}_{ \pm}-\mathrm{ee}_{ \pm} \mathrm{B}_{ \pm} /\left(1+\mathrm{t}^{2}\right)\left(1 \mp \mathrm{i} \gamma_{0} \mathrm{ux}\right)$,

where the terms $\mathrm{A}_{+}$and $\mathrm{B}_{+}$are given by

$\dot{A}_{+}=\beta_{0} /\left(1+t^{2}\right)\left(1 \mp \mathrm{i} \gamma_{0} u x\right)-\beta_{0}^{3} /\left(1+\beta_{0}^{2}+\gamma_{0}^{2} x^{2}\right)$ $-\gamma_{0} \mathrm{~F}^{2} /\left(1+\mathrm{t}^{2}\right)^{2}\left(1 \mp \mathrm{i} \gamma_{0} \mathrm{ux}\right)^{2}$,

$\mathbf{B}_{ \pm}=\beta_{0}-\gamma_{0} \mathrm{~d}-\gamma_{0} \mathrm{~F}^{2} /\left(1+\mathrm{t}^{2}\right)\left(1 \mp \mathrm{i} \gamma_{0} \mathrm{ux}\right)$.

It may be verified that we have

$$
\begin{aligned}
& \mathrm{A}_{ \pm}= \beta_{0}\left[\left(1-\beta_{0}^{2} t^{2}\right) /\left(1+\mathrm{t}^{2}\right)+\mathrm{x}^{2} \beta_{0}^{2} \gamma_{0}^{2} /\left(1+\beta_{0}^{2}+\gamma_{0}^{2}\right)\right] /\left(1+\beta_{0}^{2}\right) \\
&+\gamma_{0} \mathrm{C}_{ \pm} \\
& \mathrm{B}_{ \pm} /\left(1+\mathrm{t}^{2}\right)\left(1 \mp i \gamma_{0} \mathrm{ux}\right)=\beta_{0} /\left(1+\mathrm{t}^{2}\right)-\gamma_{0} \mathrm{D}_{ \pm},
\end{aligned}
$$

where the terms $C_{+}$and $D_{+}$are given by

$$
\begin{aligned}
C_{ \pm}= & {\left[\beta_{0}^{3} y_{0}^{3} /\left(1+\beta_{0}^{2}\right)\left(1+\beta_{0}^{2}+\gamma_{0}^{2}\right)\right] \mathrm{x}^{2}\left(1-\mathrm{x}^{2}\right) /\left(1+\beta_{0}^{2}+\gamma_{0}^{2} \mathrm{x}^{2}\right) } \\
- & {\left[\beta_{0} y_{0} \mathrm{t}^{2} \mathrm{x}^{2}+\mathrm{F}^{2}\left(1+\mathrm{t}^{2}-\gamma_{0}^{2} \mathrm{t}^{2} \mathrm{x}^{2}\right) /\left(1+\mathrm{t}^{2}+\gamma_{0}^{2} \mathrm{t}^{2} \mathrm{x}^{2}\right)\right.} \\
& \left.\mp \mathrm{it}\left(1+\mathrm{t}^{2}\right)^{1 / 2}\left\{\beta_{0}-2 \mathrm{~F}^{2} \gamma_{0} /\left(1+\mathrm{t}^{2}+\gamma_{0}^{2} \mathrm{t}^{2} \mathrm{x}^{2}\right)\right\} \mathrm{x}\right] \\
& /\left(1+\mathrm{t}^{2}\right)\left(1+\mathrm{t}^{2}+\gamma_{0}^{2} \mathrm{t}^{2} \mathrm{x}^{2}\right),
\end{aligned}
$$

$$
\left(1+t^{2}\right)\left(1+t^{2}+\gamma_{0}^{2} t^{2} x^{2}\right) D_{ \pm}=\beta_{0} y_{0} t^{2} x^{2}+d\left(1+t^{2}\right)
$$$$
+\mathrm{F}^{2}\left(1+\mathrm{t}^{2}-\gamma_{0}^{2} \mathrm{t}^{2} \mathrm{x}^{2}\right) /\left(1+\mathrm{t}^{2}+\gamma_{0}^{2} \mathrm{t}^{2} \mathrm{x}^{2}\right)
$$$$
\mp \mathrm{i}\left(1+\mathrm{t}^{2}\right)^{1 / 2}\left[\beta_{0}-\gamma_{0} \mathrm{~d}-2 \mathrm{~F}^{2} \gamma_{0} /\left(1+\mathrm{t}^{2}+\gamma_{0}^{2} \mathrm{t}^{2} \mathrm{x}^{2}\right)\right] \mathrm{x} \text {. }
$$

Equation (50) yields

$\exp \left[i v^{2}\left(1+t^{2}\right)^{1 / 2}\left(1 \mp \beta_{0} t\right) x\right] e_{+}=$

$\exp \left[i v^{2}\left(1+t^{2}\right)^{1 / 2}\left(1 \mp \beta_{d} t\right) x\right]$,

where $\beta_{d}$ is defined as

$\beta_{\mathrm{d}}=\beta_{0}-\mathrm{d} \gamma_{0}=\tan \beta-\mathrm{dtan} \gamma$

as may be obtained from equations $(42 a, b)$. By using equations (55), (51), (52a,b) and (44) into equation (48) we may obtain

$\exp \left[i v^{2}\left(1+t^{2}\right)^{1 / 2}\right] K_{0}^{ \pm}(t)=$

$\beta_{0}\left[\left(1-\beta_{0}^{2} t^{2}\right)\left(1+t^{2}\right)^{-1} I_{0}^{ \pm}\left(t ; \beta_{0}\right)+\beta_{0}^{2} \gamma_{0}^{2}\left(1+\beta_{0}^{2}+\gamma_{0}^{2}\right)^{-1} I_{I}^{ \pm}\left(t ; \beta_{0}\right)\right.$

$\left.-\left(1+\beta_{0}^{2}\right) \exp \left\{-v^{2} \mathrm{~d}\left(1+\mathrm{t}^{2}\right)\right\}\left(1+\mathrm{t}^{2}\right)^{-1} I_{0}^{ \pm}\left(\mathrm{t} ; \beta_{\mathrm{d}}\right)\right] /\left(1+\beta_{0}^{2}\right)$

$+\gamma_{0}\left[\mathrm{~J}_{ \pm}\left(\mathrm{t} ; \beta_{0}, \mathrm{C}_{ \pm}\right)+\exp \left\{-\nu^{2} \mathrm{~d}\left(1+\mathrm{t}^{2}\right)\right\} \mathrm{J}_{ \pm}\left(\mathrm{t} ; \beta_{\mathrm{d}}, \mathrm{D}_{ \pm}\right)\right]$, 
precisely $I_{n}^{ \pm}(t ; \beta, v)$ and $J_{ \pm}(t ; \beta, A, v)$, are defined in terms of the integrals

$I_{n}^{ \pm}(t ; \beta)=\nu^{2} \int_{0}^{1} \exp \left[i^{2}\left(1+t^{2}\right)^{1 / 2}(1 \mp \beta t) x\right] x^{2 n} d x$,

$J_{ \pm}(t ; \beta, A)=\nu^{2} \int_{0}^{1} \exp \left[i v^{2}\left(1+t^{2}\right)^{1 / 2}(1 \mp \beta t) x\right] A(x) d x .(58 b)$

The integrals $I_{n}^{ \pm}(t ; \beta)$ can be evaluated analytically, with the result

$I_{0}^{ \pm}(t ; \beta)=i\left(1-E_{ \pm}\right) / \sigma_{ \pm}$,

$I_{1}^{ \pm}(t ; \beta)=-i\left[\left(1+2 \mathrm{iF}^{2} / \sigma_{ \pm}\right) \mathrm{E}_{ \pm}+2 \mathrm{~F}^{4}\left(1-\mathrm{E}_{ \pm}\right) / o_{ \pm}^{2}\right] / o_{ \pm}$,

where $\sigma_{ \pm}$and $\mathrm{E}_{ \pm}$are defined as

$o_{ \pm}=\left(1+t^{2}\right)^{1 / 2}(1 \mp \beta \mathrm{t}), \quad \mathrm{E}_{ \pm}=\exp \left(i v^{2} \sigma_{ \pm}\right)$.

$(59 c, d)$

Expressions $(59 a, b)$ for $I_{0}^{+}(t ; \beta)$ and $I_{1}^{+}(t ; \beta)$ are not valid in the special case when we have $t=1 / \beta$, for which expressions $(59 \mathrm{a}, \mathrm{b})$ become

$I_{0}^{+}(1 / \beta ; \beta)=v^{2}, \quad I_{1}^{+}(1 / \beta ; \beta)=\nu^{2} / 3$.

(59e,f)

The integrals $\mathrm{J}_{+}$defined by equation $(58 \mathrm{~b})$, where the amplitude function $\mathbf{A}(\mathrm{x})$ takes the form of $\mathrm{C}_{ \pm}$or $\mathrm{D}_{ \pm}$ specified in equations $(53 \mathrm{a}, \mathrm{b})$, cannot be evaluated analytically. These integrals were then evaluated numerically by dividing the integration range $0 \leqslant x \leqslant 1$ into $N$ segments of equal length and using piecewise quadratic approximations for the amplitude function $\mathbf{A}(\mathrm{x})$ within each segment. In this manner, we may obtain

$\sigma_{ \pm} J_{ \pm}(t ; \beta, A) \simeq \sum_{j=1}^{N}\left(\varepsilon_{ \pm}\right)^{j-1}\left[i\left(A_{j}-\varepsilon_{ \pm} A_{j+1}\right)\right.$

$+\left(\mathrm{NF}^{2} / \sigma_{ \pm}\right)\left(\varepsilon_{ \pm}-1\right)\left(\mathrm{A}_{\mathrm{j}+1}-\mathrm{A}_{\mathrm{j}}\right)+\left(2 \mathrm{NF}^{2} / \sigma_{ \pm}\right)$

$\left.\left\{1+\varepsilon_{ \pm}+\mathrm{i}\left(2 \mathrm{NF}^{2} / \sigma_{ \pm}\right)\left(\varepsilon_{ \pm}-1\right)\right\}\left(\mathrm{A}_{\mathrm{j}}+\mathrm{A}_{\mathrm{j}+1}-2 \mathrm{~A}_{\mathrm{j}+1 / 2}\right)\right]$,

where $\sigma_{ \pm}$is given by equation $(59 \mathrm{c}), \varepsilon_{ \pm}$is defined as

$\varepsilon_{ \pm}=\exp \left(\mathrm{iv}^{2} a_{ \pm} / \mathrm{N}\right)$,

and $A_{j}, A_{j+1}, A_{j+1 / 2}$ represent the values of the amplitude function $A(x)$ at the points $x_{j}=(j-1) / N, x_{j+1}$ $=j / N$, and $x_{j+1 / 2}=(j-1 / 2) / N$, respectively. Expression $(60)$ for $J_{+}(t ; \beta, A)$ is not valid in the special case when $t$

$=1 / \beta$, for which we have

$6 \mathrm{NJ}+(1 / \beta ; \beta, A) \simeq \sum_{j=1}^{N} A_{j}+A_{j+1}+4 A_{j+1 / 2}$.

In summary, the zeroth-order slender-ship

approximation $\mathrm{K}_{0}(\mathrm{t})$ is determined by equations (46), (57),

$(59 \mathrm{a}-\mathrm{f}),(60),(60 \mathrm{a}, \mathrm{b}),(42 \mathrm{a}, \mathrm{b})$ and $(56)$. In the limiting case

$\gamma_{0}=0$, these equations yield

$\mathrm{K}_{0}(\mathrm{t})=4 \beta_{0}\left(1+\mathrm{t}^{2}\right)^{-3 / 2}\left[\left(1-\beta_{0}^{2} \mathrm{t}^{2}\right)\left(1+\beta_{0}^{2}\right)^{-1}\right.$

$\left.-\exp \left\{-v^{2} \mathrm{~d}\left(1+\mathrm{t}^{2}\right)\right\}\right](\mathrm{R}-\mathrm{i} \mathrm{I}) /\left(1-\beta_{0}^{2} \mathrm{t}^{2}\right)$,

where the terms $\mathrm{R}$ and $\mathrm{I}$ are given by

$R=\sin \left[v^{2}\left(1+t^{2}\right)^{1 / 2} / 2\right] \cos \left[v^{2}\left(1+t^{2}\right)^{1 / 2} / 2\right]$

$-\beta_{0} t \sin \left[v^{2} \beta_{0} t\left(1+t^{2}\right)^{1 / 2} / 2\right] \cos \left[v^{2} \beta_{0} t\left(1+t^{2}\right)^{1 / 2} / 2\right]$,

$I=\sin ^{2}\left[\nu^{2}\left(1+t^{2}\right)^{1 / 2} / 2\right]-\sin ^{2}\left[\nu^{2} \beta_{0} t\left(1+t^{2}\right)^{1 / 2} / 2\right]$.
In the thin-ship limit $\beta_{0} \rightarrow 0$, equations (61) and $(61 \mathrm{a}, \mathrm{b})$ yield

$K_{0}(t) \sim 4 \beta_{0}\left(1+t^{2}\right)^{-3 / 2}\left[1-\exp \left\{-v^{2} d\left(1+t^{2}\right)\right\}\right]$ $\sin \left[v^{2}\left(1+t^{2}\right)^{1 / 2} / 2\right] \exp \left[-i v^{2}\left(1+t^{2}\right)^{1 / 2} / 2\right]$ as $\beta_{0} \rightarrow 0$.

The thin-ship limit (62) of the zeroth-order slender-ship approximation $\mathbf{K}_{0}(t)$ may be seen to be identical to the Michell thin-ship approximation (43) in the particular case $\gamma_{0}=0$. However, the thin-ship limit (62) of the slendership approximation (61) is not uniformly valid in the limit $t \rightarrow \infty$; indeed, equations $(61)$ and $(61 \mathrm{a}, \mathrm{b})$ yield equation $(62)$ in the limit $\beta_{0} t \rightarrow 0$. Equation (62) yields $\left|K_{0}(t)\right| \sim 4 \beta_{0}\left|\sin \left(v^{2} t / 2\right)\right| / t^{3}$ for $1 \ll t \ll 1 / \beta_{0}$.

More generally, equation (61) yields

$\left|\mathrm{K}_{0}(t)\right| \sim 4 \beta_{0}|\mathbf{R}-\mathrm{il}| /\left(1+\beta_{0}^{2}\right) \mathrm{t}^{3}$ as $\mathrm{t} \rightarrow \infty$.

Equations $(61 \mathrm{a}, \mathrm{b})$ show that $|\mathrm{R}-\mathrm{il}| \sim \beta_{0} \mathrm{t}\left|\sin \left(\nu^{2} \beta_{0} \mathrm{t}^{2}\right)\right| / 2$ if $\beta_{0} t \gg 1$. We then have

$\left|K_{0}(t)\right| \sim 2 \beta_{0}^{2}\left|\sin \left(v^{2} \beta_{0} t^{2}\right)\right| /\left(1+\beta_{0}^{2}\right) t^{2}$ for $t \gg 1 / \beta_{0}$.

In the limit $t \rightarrow \infty$, equations (57) and (59a-d) yield

$\exp \left(i v^{2} t\right) K_{0}^{ \pm}(t) \sim \beta_{0}\left[\left(1-\beta_{0}^{2} t^{2}\right) I_{0}^{ \pm}\left(t ; \beta_{0}\right)\right.$

$\left.+\gamma_{0}^{2}\left(1+\beta_{0}^{2}+\gamma_{0}^{2}\right)^{-1} \beta_{0}^{2} t^{2} I_{1}^{ \pm}\left(t ; \beta_{0}\right)\right] /\left(1+\beta_{0}^{2}\right) t^{2}$ as $\mathrm{t} \rightarrow \infty$,

where we have

$\mathrm{I}_{0}^{ \pm}\left(\mathrm{t} ; \beta_{0}\right) \sim \mathrm{i}\left[1-\exp \left\{\mathrm{iv}^{2} \mathrm{t}\left(1 \mp \beta_{0} \mathrm{t}\right)\right\}\right] /\left(1 \mp \beta_{0} \mathrm{t}\right) \mathrm{t}$ as $\mathrm{t} \rightarrow \infty$,

$I_{1}^{ \pm}\left(t ; \beta_{0}\right) \sim-i \exp \left\{i v^{2} t\left(1 \mp \beta_{0} t\right)\right\} /\left(1 \mp \beta_{0} t\right) t$ as $t \rightarrow \infty$.

We then have

$\left|\mathrm{K}_{0}(\mathrm{t})\right| \sim 2 \beta_{0}|N| /\left(1+\beta_{0}^{2}\right)\left|1-\beta_{0}^{2} \mathrm{t}^{2}\right| \mathrm{t}^{3}$ as $\mathrm{t} \rightarrow \infty$,

where the term $\mathrm{N}$ is given by

$N=\left(1-\beta_{0}^{2} t^{2}\right)\left[\sin \left(v^{2} t\right)+i \cos \left(v^{2} t\right)\right]$

$-\left(1-o^{2} \beta_{0}^{2} t^{2}\right)\left[\beta_{0} t \sin \left(v^{2} \beta_{0} t^{2}\right)+i \cos \left(v^{2} \beta_{0} t^{2}\right)\right]$,

with $a^{2}$ defined as

$\sigma^{2}=\left(1+\beta_{0}^{2}\right) /\left(1+\beta_{0}^{2}+\gamma_{0}^{2}\right)$.

We may then obtain

$\left|K_{0}(t)\right| \sim 4 \beta_{0}\left|\sin \left(\nu^{2} t / 2\right)\right| /\left(1+\beta_{0}^{2}\right) t^{3}$ for

$1 \ll \mathrm{t} \ll \mathrm{F} /\left(\beta_{0}\right)^{1 / 2}$,

$\left|\mathrm{K}_{0}(\mathrm{t})\right| \sim 2 \beta_{0}^{2}\left|\sin \left(\nu^{2} \beta_{0} \mathrm{t}^{2}\right)\right| /\left(1+\beta_{0}^{2}+\gamma_{0}^{2}\right) \mathrm{t}^{2}$ for $\mathrm{t} \gg 1 / \beta_{0}$

Equations $(67 \mathrm{a}, \mathrm{b})$ are identical to equations $(63)$ and $(65)$

in the limits $\beta_{0} \rightarrow 0$ and $\gamma_{0} \rightarrow 0$, respectively. More

generally, equation (66a) yields the following upper bound for the term $|N|$ in equation (66)

$\begin{aligned}|N|^{2} & \leqslant\left(1-\beta_{0}^{2} t^{2}\right)^{2}+\left(1+\beta_{0}^{2} t^{2}\right)\left(1-\sigma^{2} \beta_{0}^{2} t^{2}\right)^{2} \\ & +2\left(1+\beta_{0} t\right)^{2}\left(1+\sigma \beta_{0} t\right)\left|\left(1-\beta_{0} t\right)\left(1-\sigma \beta_{0} t\right)\right| .\end{aligned}$

Equations $(67 a, b)$ thus show that in the limit $t \rightarrow \infty$ we have $\left|K_{0}(t)\right| \sim 1 / t^{3}$ for $1 \ll t \ll F /\left(\beta_{0}\right)^{1 / 2}$, that is for moderately large values of $t$, and $\left|K_{0}(t)\right| \sim 1 / t^{2}$ for $1 / \beta_{0}$ $\ll$ t, that is for very large values of $t$. The asymptotic approximations $(67 \mathrm{a}, \mathrm{b})$ and $(45)$ show that the Michell approximation $\mathrm{K}_{\mathrm{M}}(\mathrm{t})$ corresponds to the thin-ship limit $\beta_{0}$ $\ll 1$ of the zeroth-order slender-ship approximation $K_{0}(t)$, 


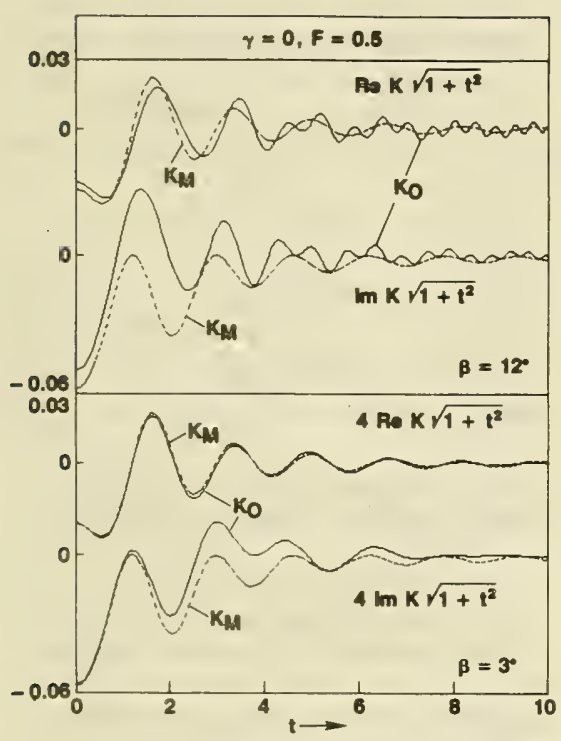

Fig. 9a - Real and Imaginary Parts of the Functions $K_{M}(t)$ and $K_{0}(t)$ for a Simple Ship Bow Form with $\beta=12^{\circ}$ (Top) and $3^{\circ}$ (Bottom), $\gamma=0$ and $F=0.5$

and that this thin-ship limit is not uniformly valid in the limit $t \rightarrow \infty$. More generally, the limiting processes $\beta_{0} \rightarrow 0$ and $\mathrm{t} \rightarrow \infty$ cannot be interchanged.

Figures $9 \mathrm{a}, \mathrm{b}$ depict the real and/or imaginary parts of the functions $\left(1+t^{2}\right)^{1 / 2} K_{M}(t)$ and $\left(1+t^{2}\right)^{1 / 2} K_{0}(t)$ for the simple bow shape defined by equations $(40 a, b)$, where the nondimensional draft $\mathrm{d}$ and the maximum flare angle $y$ are taken equal to $d=0.1$ and $\gamma=0$, and four values of the half-entrance-angle $\beta$ are considered, namely $\beta=$ $12^{\circ}, 3^{\circ}, 1^{\circ}$ and $20^{\prime}$. The Froude number based on the length of the bow region is taken equal to $F=0.5$ in the numerical results presented in figures $9 \mathrm{a}, \mathrm{b}$ and in figures 10 and 11 considered further on. The values of $1 / \beta_{0}=$ $1 / \tan \beta$ corresponding to the values of $\beta$ equal to $12^{\circ}, 3^{\circ}$, $1^{\circ}$ and $20^{\prime}$ are approximately equal to $4.7,19,57$ and 172 , respectively. The functions $\left(1+t^{2}\right)^{1 / 2} K_{M}(t)$ and $\left(1+t^{2}\right)^{1 / 2} K_{0}(t)$ are depicted for $0 \leqslant t \leqslant 10$ in figures $9 a, b$. Differences between the approximations $\mathbf{K}_{\mathrm{M}}$ and $\mathrm{K}_{0}$, especially their imaginary parts, can be seen to be substantial in figure $9 \mathrm{a}$ corresponding to $\beta=12^{\circ}$ and $3^{\circ}$.

Figure $9 \mathrm{~b}$ shows that differences between the imaginary parts of the functions $K_{M}$ and $K_{0}$ remain appreciable even

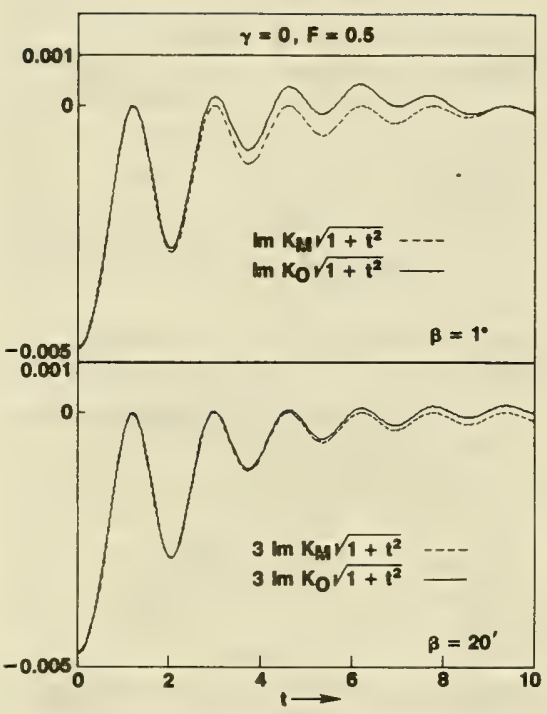

Fig. $9 \mathrm{~b}-$ Real and Imaginary Parts of the Functions $K_{M}(t)$ and $K_{0}(t)$ for a Simple Ship Bow Form with $\beta=1^{\circ}$ (Top) and 20' (Bottom), $\gamma=0$ and $F=0.5$

for values of $\beta$ equal to $1^{\circ}$ and $20^{\prime}$, which are quite small, and for values of that are much smaller than $1 / \beta_{0}$.

The top and bottom parts of figure 10 depict the real and imaginary parts of the functions $\left(1+t^{2}\right)^{1 / 2} K_{M}(t)$ and $\left(1+t^{2}\right)^{1 / 2} K_{0}(t)$, respectively, for the previouslyconsidered simple bow shape with $\mathrm{d}=0.1, \beta=12^{\circ}, \mathrm{F}$ $=0.5$ and for two values of the flare angle, namely for $y$ $=0$ and $45^{\circ}$. The top part of the figure shows that differences between the curves corresponding to $\gamma=0$ and $\gamma=45^{\circ}$ are faily small, and are appreciable only for small values of $t$, for the Michell thin-ship approximation $\mathrm{K}_{\mathrm{M}}$. In particular, the asymptotic approximation $\mathrm{K}_{\mathrm{M}}(\mathrm{t}) \sim 4 \beta_{0} \sin \left(v^{2} \mathrm{t} / 2\right) \exp \left(-\mathrm{i} v^{2} \mathrm{t} / 2\right) / \mathrm{t}^{3}$ as $\mathrm{t} \rightarrow \infty$, which may be obtained from equations (43) and (44), is independent of $\gamma$. The bottom part of figure 10 shows differences between the curves corresponding to $\gamma=0$ and $\gamma=45^{\circ}$ for the slender-ship approximation $\mathrm{K}_{0}$ that are significantly larger than those for the Michell approximation $K_{M}$, especially for intermediate values of $t$ in the vicinity of $t=1 / \tan \beta \simeq 4.7$. The flare angle $y$ thus has a pronounced effect upon the behavior of the 


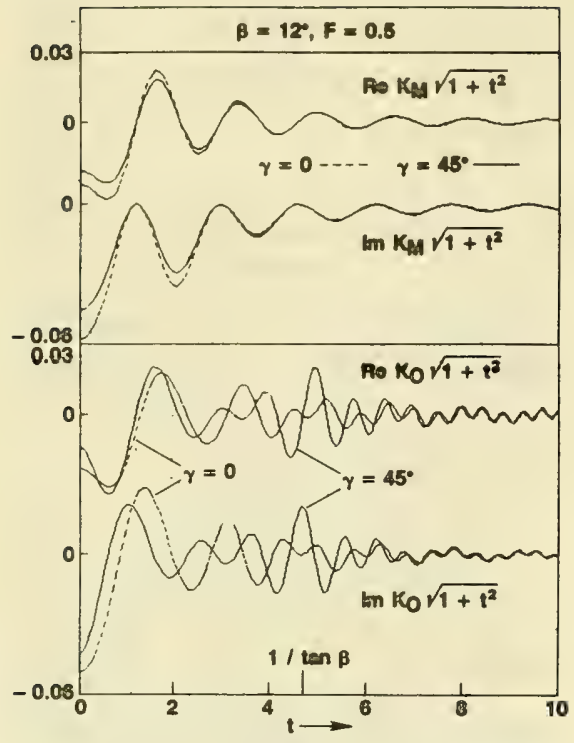

Fig. 10 - Real and Imaginary Parts of the Functions $K_{M}(t)$ and $K_{0}(t)$ for a Simple Ship Bow Form with $\gamma=0$ and $45^{\circ}, \beta=12^{\circ}$ and $F=0.5$

slender-ship approximation $\mathrm{K}_{0}(\mathrm{t})$ for values of $\mathrm{t}$ in the neighborhood of $1 / \tan \beta$. The influence of $\gamma$ upon $\left|K_{0}(t)\right|$ for large values of $t$ is explicitly indicated by the asymptotic approximations $(67 a, b)$.

Finally, figure 11 depicts the real and imaginary parts of the functions $\left(1+t^{2}\right)^{1 / 2} \mathrm{~K}_{M}(\mathrm{t})$ and $\left(1+t^{2}\right)^{1 / 2} \mathrm{~K}_{0}(\mathrm{t})$ for the simple bow shape depicted in figure 8 , for which we have $d=0.1, \beta=12^{\circ}$ and $\gamma=45^{\circ}$, at a value of the Froude number $F$ equal to 0.5. Differences between the approximations $K_{M}$ and $K_{0}$ may be seen to be quite substantial. In particular, the function $\left(1+t^{2}\right)^{1 / 2} K_{0}(t)$ has a peak at $t=1 / \tan \beta=1 / \beta_{0}$.

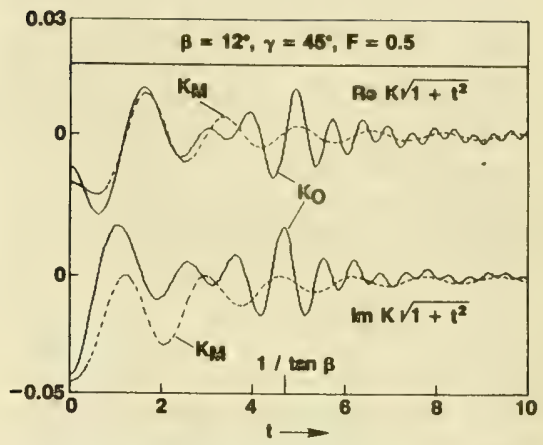

Fig. 11 - Real (Top) and Imaginary (Bottom) Parts of the Functions $K_{M}(t)$ and $K_{0}(t)$ for a Simple Ship Bow

Form with $\beta=12^{\circ}, y=45^{\circ}$ and $F=0.5$

\section{A SIMPLE TEST CASE: THE FAR-FIELD KELVIN WAKE}

The expressions for the far-field wave-amplitude function $\mathrm{K}(\mathrm{t})$ obtained in the foregoing section for a simple bow shape and for two simple approximations to the function $K(t)$, namely the Michell thin-ship approximation $\mathrm{K}_{\mathrm{M}}$ and the zeroth-order slender-ship approximation $\mathrm{K}_{0}$, may now be used into the previously determined asymptotic approximations for the far-field Kelvin wake.

Far behind the ship, that is for $x \rightarrow-\infty$, the functions $\phi_{\mathrm{k}}(\mathrm{x}, \alpha)$, where $0 \leqslant \mathrm{k} \leqslant 4$, defined by equations (17), (18), (19) and (20a-e) are given by the asymptotic approximation (23). The real and imaginary parts of the amplitude functions $\mathrm{A}_{\mathrm{k}}^{-}(\alpha)$ and $\mathrm{A}_{\mathrm{k}}^{+}(\alpha)$ in this asymptotic approximation, given by equation (24a), are depicted in figures $12 a$ and $b, 13 a$ and $b$ and 14 for $0 \leqslant \alpha \leqslant 1 / 2^{3 / 2}$. More precisely, figures $12 \mathrm{a}, \mathrm{b}$ and $13 \mathrm{a}, \mathrm{b}$ represent the amplitude functions $A_{k}^{-}$and $A_{k}^{+}$for $k=0,1,2,3$ associated with the potential $\phi$ and its derivatives $\phi_{x}, \phi_{y}$ and $\phi_{\mathrm{xx}}$, and correspond to the approximations $\mathrm{K}_{\mathrm{M}}$ and 
$\mathrm{K}_{0}$, respectively. Figure 14 depicts the amplitude functions $\mathrm{A}_{4}^{-}$and $\mathrm{A}_{4}^{+}$corresponding to $\phi_{x y}$ for the approximations $K_{M}$ and $K_{0}$.

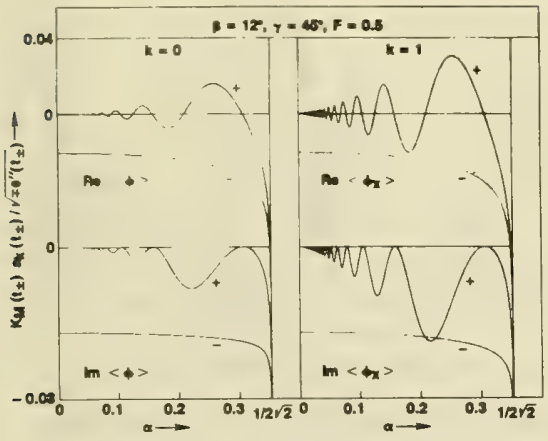

Fig. 12a - Real (Top) and Imaginary (Bottom) Parts of the Functions $K_{+} a_{k}^{ \pm} /\left(\mp \theta_{ \pm}^{\prime \prime}\right)^{1 / 2}$ for $k=0$ (Left) and 1 (Right), $\mathrm{K}=\mathrm{K}_{\mathrm{M}}$ (Michell Thin-Ship Approximation) and a Simple Ship Bow Form with $\beta=12^{\circ}, \gamma=45^{\circ}$ and $\mathrm{F}=0.5$

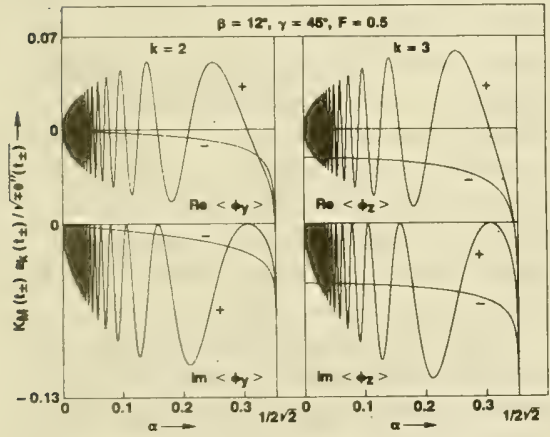

Fig. 12b - Real (Top) and Imaginary (Bottom) Parts of the Functions $K_{ \pm} a_{k}^{ \pm} /\left(\mp \theta_{ \pm}^{\prime \prime}\right)^{1 / 2}$ for $k=2$ (Left) and 3 (Right), $\mathrm{K}=\mathrm{K}_{\mathrm{M}}$ (Michell Thin-Ship Approximation) and a Simple Ship Bow Form with $\beta=12^{\circ}, \gamma=45^{\circ}$ and

$$
\mathrm{F}=0.5
$$

It may be seen from figures $12 a$ and $b, 13 a$ and $b$ and 14 that the amplitude functions $\mathrm{A}_{\mathrm{k}}^{-}(\alpha)$ and $\mathrm{A}_{\mathrm{k}}^{+}(\alpha)$ become unbounded in the limit $a \rightarrow 1 / 2^{3 / 2}$. This singularity at $\alpha=1 / 2^{3 / 2}$ stems from the fact that the asymptotic approximation (23) is not uniformly valid in the limit $\alpha \rightarrow 1 / 2^{3 / 2}$, as was already noted. The amplitude functions $\mathrm{A}_{\mathrm{k}}^{+}(\alpha)$ corresponding to the system of divergent waves in the Kelvin wake also become unbounded in the limit $a \rightarrow 0$ in figures $13 b$ and 14 corresponding to $k=2$

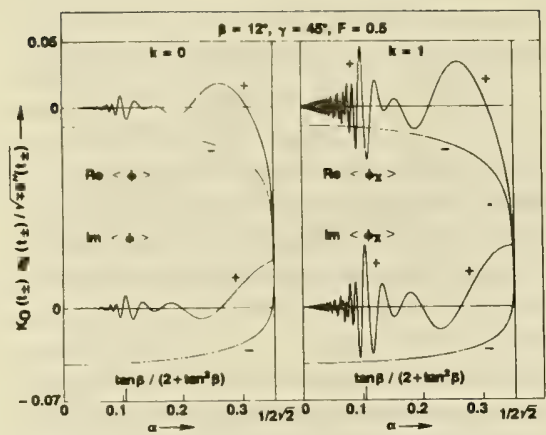

Fig. 13a - Real (Top) and Imaginary (Bottom) Parts of the Functions $K_{ \pm} a_{k}^{ \pm} /\left(\mp \theta_{ \pm}^{\prime \prime}\right)^{3 / 2}$ for $k=0$ (Left) and 1

(Right), $\mathbf{K}=\mathbf{K}_{0}$ (Zeroth-Order Slender-Ship

Approximation) and a Simple Ship Bow Form with $\beta=12^{\circ}, \gamma=45^{\circ}$ and $F=0.5$

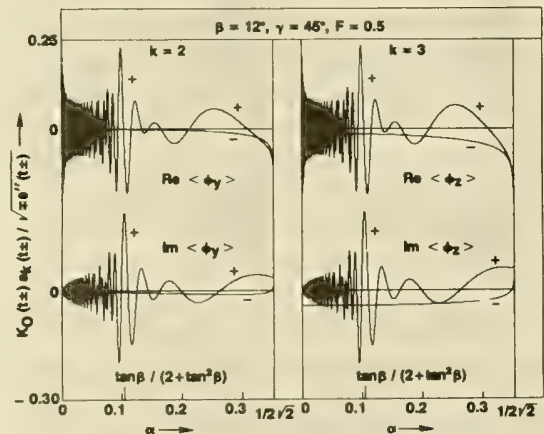

Fig. 13b - Real (Top) and Imaginary (Bottom) Parts of the Functions $K_{ \pm} a_{k}^{ \pm} /\left(\mp \Theta_{ \pm}^{\prime \prime}\right)^{1 / 2}$ for $k=2$ (Left) and 3

(Right), $\mathrm{K}=\mathrm{K}_{0}$ (Zeroth-Order Slender-Ship

Approximation) and a Simple Ship Bow Form with $\beta=12^{\circ}, \gamma=45^{\circ}$ and $F=0.5$

and 3 for the slender-ship approximation $K_{0}$ and to $k=4$ for both approximations $K_{M}$ and $K_{0}$, respectively. This singularity at $a=0$ illustrates the previously-noted conclusion that the asymptotic approximation (23) is not uniformly valid in the vicinity of the track of the ship a $=0$ if condition (27) is not satisfied. Equations (20a-e) show that we have $a_{0} \sim 1, a_{1} \sim t, a_{2} \sim t^{2}, a_{3} \sim t^{2}$ and $a_{4} \sim t^{3}$ as $t \rightarrow \infty$, and equations (45) and (67b) yield $\left|K_{M}\right| \sim 1 / t^{3}$ and $\left|K_{0}\right| \sim 1 / t^{2}$ as $t \rightarrow \infty$, respectively. Condition (27) therefore is not satisfied for $k \geqslant 4$ and $k$ $\geqslant 2$ for the approximations $K_{M}$ and $K_{0}$, respectively. Condition (27) however is satisfied for $k=0$ and 1 , 
corresponding to the potential $\phi$ and the free-surface elevation $\phi_{x}$, for both the approximations $K_{M}$ and $K_{0}$.

Figures $12 \mathrm{a}$ and $13 \mathrm{a}$ show that the amplitude functions $A_{0}^{-}$and $A_{1}^{-}$, corresponding to the transverse waves in the Kelvin wake, generally are larger in magnitude than the amplitude functions $\mathrm{A}_{0}^{+}$and $\mathrm{A}_{1}^{+}$ corresponding to the divergent waves, whereas the reverse may generally be seen to hold in figures $12 \mathrm{~b}$ and $13 \mathrm{~b}$ for the amplitude functions $\mathbf{A}_{2}^{ \pm}$and $\mathbf{A}_{3}^{ \pm}$. The relative importance of the divergent waves with respect to the transverse waves thus increases with $k$. Indeed, the transverse-wave amplitude function $\mathbf{A}_{4}^{-}(\alpha)$ is hardly visible on the scale of the divergent-wave-amplitude function $\mathrm{A}_{4}^{+}(a)$ used in figure 14 .

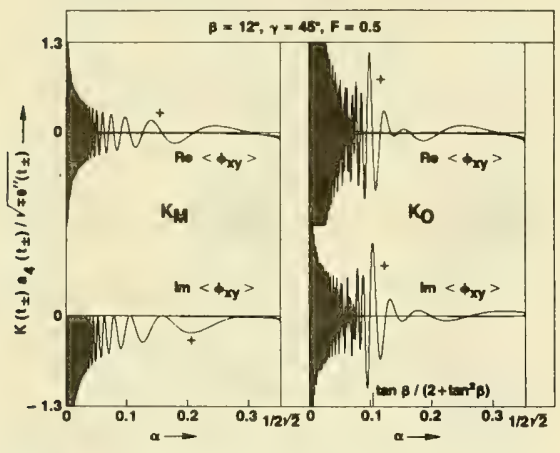

Fig. 14 - Real (Top) and Imaginary (Bottom) Parts of the Functions $K_{ \pm} a_{4}^{ \pm} /\left(\mp \Theta_{ \pm}^{\prime \prime}\right)^{1 / 2}$ for $K_{M}$ (Left) and $K_{O}$

(Right) and a Simple Ship Bow Form with $\beta=12^{\circ}$,

$$
y=45^{\circ} \text { and } F=0.5
$$

The divergent-wave-amplitude functions $\mathrm{A}_{\mathrm{k}}^{+}(\alpha)$ associated with the approximation $\mathrm{K}_{0}$ are most notably different from the corresponding functions $\mathrm{A}_{\mathrm{k}}^{+}(\alpha)$ associated with the Michell approximation $K_{M}$ for values of $\alpha$ in the vicinity of $\alpha=0$, as was already noted, and of $\alpha=\tan \beta /\left(2+\tan ^{2} \beta\right)$. In the vicinity of this value of $\alpha$, the divergent-wave-amplitude functions $\mathrm{A}_{k}^{+}(\alpha)$ associated with the approximation $K_{0}$ exhibit a peak, which is quite pronounced for $k \geqslant 1$. The foregoing particular value of $\alpha$ corresponds to the special case when the point of stationary phase $t_{+}$, defined by equation (12), is equal to the value $1 / \beta_{0}=1 / \tan \beta$ for which the function $K_{0}(t)$ displays a peak, as may be seen from figures 10 and 11 .

Figure 15 depicts the amplitude functions $(2 / \pi)^{1 / 2} A_{1}^{-}$and $(2 / \pi)^{1 / 2} A_{1}^{+}$that are associated with the free-surface elevation far behind the ship, as is specifically

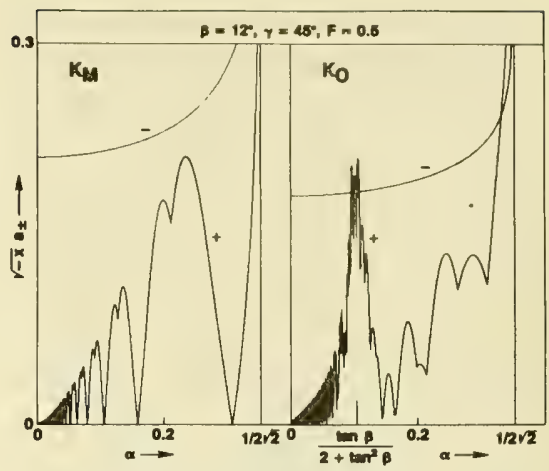

Fig. 15 - Amplitude of the Transverse ( - ) and Divergent $(+)$ Waves in the Kelvin Wake for a Simple Ship Bow Form, with $\beta=12^{\circ}, \gamma=45^{\circ}$ and $F=0.5$, in the Michell Thin-Ship Approximation (Left) and the

Zeroth-Order Slender-Ship Approximation (Right)

indicated in equation (28), for the previously-considered ship bow shape. It may be seen that the amplitude $\mathrm{a}_{+}(\alpha)$ of the divergent waves in the Kelvin wake vanishes as $\alpha \rightarrow$ 0 , that is at the track of the ship, and is generally smaller than the amplitude a_( $\alpha)$ of the transverse waves; this is especially true in the vicinity of the track of the ship. Differences between the wave-amplitude functions $\mathrm{a}_{ \pm}(\alpha)$ corresponding to the approximations $\mathrm{K}_{\mathrm{M}}(\mathrm{t})$ and $\mathrm{K}_{0}(\mathrm{t})$ are particularly striking for the amplitude $\mathrm{a}_{+}(a)$ of the divergent waves in the vicinity of $\alpha=\tan \beta /\left(2+\tan ^{2} \beta\right)$, where the function $a_{+}(\alpha)$ associated with the approximation $\mathrm{K}_{0}(\mathrm{t})$ exhibits a sharp peak.

Figure 16 depicts the wave-steepness functions $s_{-}(\alpha)$ and $s_{+}(\alpha)$, which correspond to the ratios of the waveamplitude functions $a_{-}(\alpha)$ and $a_{+}(\alpha)$ depicted in figure 15 over the wavelength functions $\lambda_{-}(\alpha)$ and $\lambda_{+}(\alpha)$ defined by equation (30a); the steepness functions $s_{ \pm}(\alpha)$ are specifically defined by equations (32) and (33). The divergent waves in the Kelvin wake may be seen to be generally steeper than the transverse waves, even though figure 15 shows the transverse waves to be larger in amplitude than the divergent waves. This is especially true near the track of the ship where the steepness of the divergent waves becomes infinitely large, even though figure 15 shows that their amplitude vanishes as $\alpha \rightarrow 0$. The divergent waves become infinitely steep at the track of the ship because the wavelength $\lambda_{+}(\alpha) \rightarrow 0$ as $\alpha \rightarrow 0$ and condition (36) is satisfied, for both the approximations $K_{M}(t)$ and $K_{0}(t)$ as may be seen from 


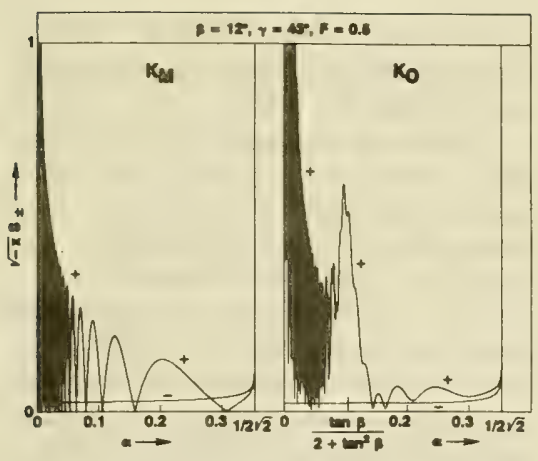

Fig. 16 - Steepness of the Transverse (-) and Divergent $(+)$ Waves in the Kelvin Wake for a Simple Ship Bow

Form, with $\beta=12^{\circ}, \gamma=45^{\circ}$ and $F=0.5$, in the

Michell Thin-Ship Approximation (Left) and the ZerothOrder Slender-Ship Approximation (Right)

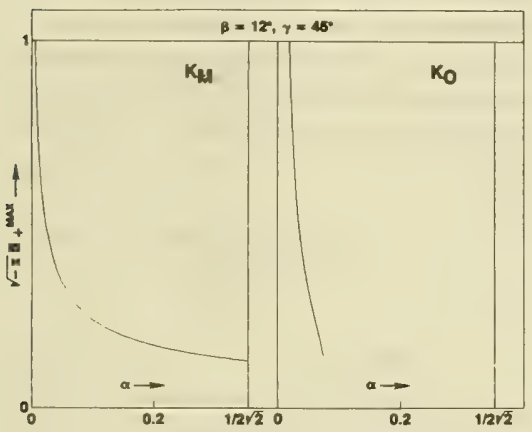

Fig. 17 - Upper Bound for the Steepness of the Divergent Waves in the Kelvin Wake for a Simple Ship Bow Form, with $\beta=12^{\circ}$ and $\gamma=45^{\circ}$, in the Michell Thin-Ship Approximation (Left) and the Zeroth-Order Slender-Ship Approximation (Right)

equations (45) and (67b). Figure 16 also shows a sharp peak in the steepness of the divergent waves at the value of $\alpha$ equal to $\tan \beta /\left(2+\tan ^{2} \beta\right)$.

The steepness $s_{+}(a)$ of the divergent waves is given by equations (32) and (33). An upper bound for the functions $s_{+}(\alpha)$ may be obtained by using an upper bound for the function $\left|K\left(t_{+}\right)\right|$in equation (32). Equation (45) yields the following upper bound for the function $\left|\mathrm{K}_{\mathrm{M}}(\mathrm{t})\right|$ corresponding to the Michell thin-ship approximation: $\left|K_{M}(t)\right| \leqslant 4 \beta_{0} / t^{3}$ as $t \rightarrow \infty$. An upper bound for the function $\left|K_{0}(t)\right|$ is given by equation (66), where the upper bound defined by equation (68) is used for the term $|\mathrm{N}|$. These upper bounds for the functions
$\left|K_{M}(t)\right|$ and $\left|K_{0}(t)\right|$ can be expressed in terms of $\alpha$ by using equation (12). The corresponding upper bounds for the steepness function $s_{+}(a)$ are depicted in figure 17. Comparison of figures 16 and 17 shows that the upper bound for the steepness of the divergent waves in the Kelvin wake depicted in figure 17 is satisfactory for all values of $\alpha$ for the Michell approximation $K_{M}$, whereas that corresponding to the slender-ship approximation $\mathrm{K}_{0}$ is satisfactory for values of a smaller than approximately half the value $\tan \beta /\left(2+\tan ^{2} \beta\right)$. In both cases, the upper bounds for the function $s_{+}(\alpha)$ depicted in figure 17 are satisfactory for the range of small values of $\alpha$ for which the steepness $s_{+}(\alpha)$ is large. It is noteworthy that these upper bounds for the steepness of the divergent waves are valid for all Froude numbers, since equations (12), (32) and (33), and the upper bounds for $\left|K_{M}(t)\right|$ and $\left|K_{0}(t)\right|$ do not involve the Froude number.

Figure 18 depicts the steepness function $s_{+}(\alpha)$ of the divergent waves corresponding to the slender-ship approximation $\mathrm{K}_{0}(\mathrm{t})$ for the simple bow shape considered previously in the two cases when the maximum flare angle $y$ is taken equal to 0 and $45^{\circ}$ (at the top and bottom halves of the figure, respectively) and for two values of the Froude number, namely for $F=0.8$ and 0.3 (on the right and left halves of the figure, respectively). Comparison of the right and left halves of figure 18 shows no appreciable difference between the values of the steepness function $s_{+}(\alpha)$ for the range of small values of $\alpha$ for which the steepness is large, in agreement with the previously-noted result that the upper bound for $s_{+}(a)$ depicted on the right side of figure 17 is independent of the Froude

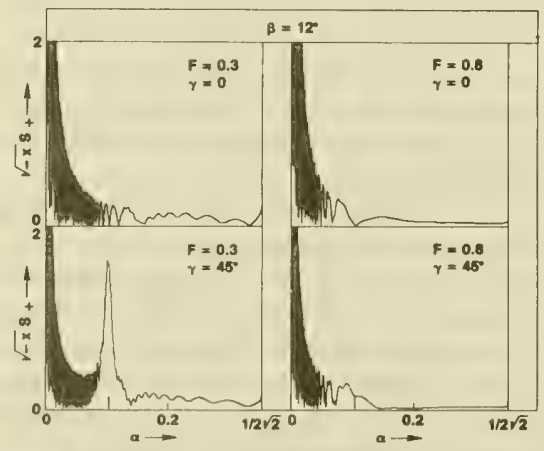

Fig. 18 - Steepness of the Divergent Waves in the Kelvin Wake for a Simple Ship Bow Form with $\beta=12^{\circ}, \gamma=0$

(Top) and $45^{\circ}$ (Bottom), and $F=0.3$ (Left) and 0.8

(Right) in the Zeroth-Order Slender-Ship Approximation 


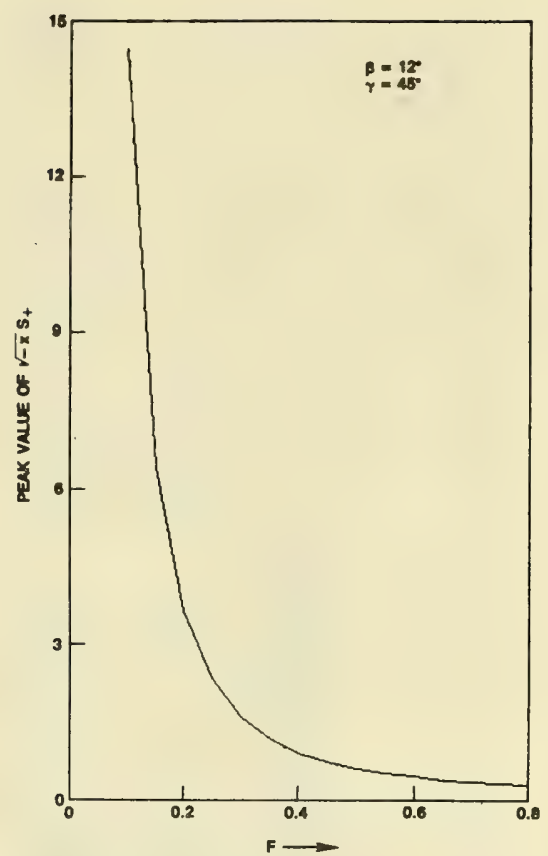

Fig. 19 - Amplitude of the Peak Value of the Steepness of the Divergent Waves in the Kelvin Wake for a Simple

Ship Bow Form with $\beta=12^{\circ}$ and $y=45^{\circ}$ in the Zeroth-Order Slender-Ship Approximation

number. Comparison of the top and bottom halves of

figure 18, corresponding to $\gamma=0$ and $45^{\circ}$ as was already noted, shows appreciable differences, especially for $\mathrm{F}=$ 0.3 for which the function $s_{+}(\alpha)$ exhibits a very pronounced peak in the case $\gamma=45^{\circ}$. No such peak is apparent in the top half of the figure for $\gamma=0$, that is in the case when the hull intersects the free surface orthogonally.

The right half of figure 16 and the bottom half of figure 18 show that the magnitude of the peak in the steepness function $s_{+}(\alpha)$ for $\alpha=\tan \beta /\left(2+\tan ^{2} \beta\right)$ strongly depends on the value of the Froude number. Specifically, the peak is very pronounced in figure 18 for $F=0.3$, fairly pronounced in figure 16 for $F=0.5$, and almost nonapparent in figure 18 for $F=0.8$. The magnitude of the peak in the steepness function, that is the value of the function $s_{+}(\alpha)$ for $\alpha=\tan \beta /\left(2+\tan ^{2} \beta\right)$, is represented in figure 19 as a function of the Froude number, which is based on the length of the bow region. This figure shows that the magnitude of the peak increases very rapidly as the Froude number decreases below a certain threshold value in the vicinity of $F=0.3$.

Figure 20 depicts the boundary of the Kelvin wake, which corresponds to $\alpha=-y / x=1 / 2^{3 / 2}$ (that is, an angle equal to approximately $19^{\circ} 28^{\prime}$ ), the line $\alpha=$ $\tan \beta /\left(2+\tan ^{2} \beta\right) \simeq \beta / 2$ (that is, an angle equal to approximately $6^{\circ}$ ) along which the steepness of the divergent waves has a peak, and the lines along which the steepness of the divergent waves is equal to $1 / 20,1 / 15$, and $1 / 7$ (shown as a chain line close to the track of the ship). The latter three lines were determined by using the upper bound for the steepness function $s_{+}(a)$ that was determined previously and depicted on the right half of figure 17. The four lines inside the Kelvin wake shown in figure 20 correspond to the zeroth-order slender-ship approximation $\mathrm{K}_{0}(\mathrm{t})$ for the simple bow shape considered previously, with $\beta=12^{\circ}, \gamma=45^{\circ}$ and $\mathrm{d}=0.1$. The three lines along which the divergent waves are steep lie much closer to the track of the ship than the lines $\alpha \simeq 6^{\circ}$ along which the steepness of the divergent waves exhibits a peak.

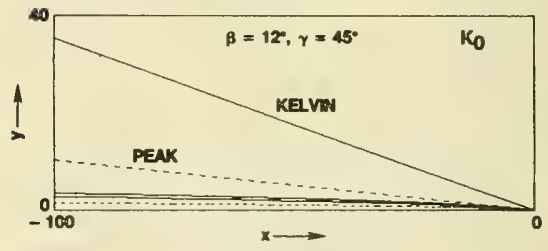

Fig. 20 - The Kelvin Cusp Line, the Line Along Which the Amplitude of the Divergent Waves Exhibits a Peak, and the Three Lines Along Which the Steepness of the Divergent Waves is Equal to $1 / 20,1 / 15$ and $1 / 7$ (Chain Line Close to the Track of the Ship) for a Simple Ship Bow Form, with $\beta=12^{\circ}$ and $\gamma=45^{\circ}$, in the ZerothOrder Slender-Ship Approximation

The three lines along which the steepness of the divergent waves is equal to $1 / 20,1 / 15$ and $1 / 7$, which are depicted in figure 20 for $0 \geqslant x \geqslant-100$ and $0 \leqslant y \leqslant 40$, are represented again in figure 21 at a distorted scale where $0 \geqslant x \geqslant-300$ and $0 \leqslant y \leqslant 7$. The corresponding constant-steepness lines predicted by the Michell thin-ship approximation are also shown in figure 21 for comparison. The latter lines were determined from equations (32) and (33) and the upper bound $\left|K_{M}(t)\right| \leqslant$ $4 \beta_{0} / t^{3}$ given by equation (45). Figure 21 shows significant differences between, the constant-steepness lines predicted 


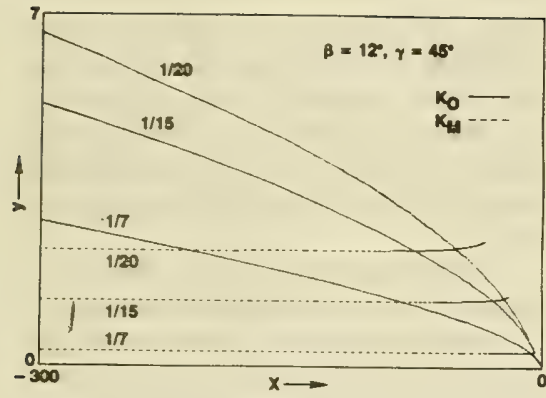

Fig. 21 - Lines Along Which the Steepness of the Divergent Waves is Equal to $1 / 20,1 / 15$ and $1 / 7$ for a Simple Ship Bow Form, with $\beta=12^{\circ}$ and $\gamma=45^{\circ}$, in the Michell Thin-Ship Approximation $(---)$ and the Zeroth-Order Slender-Ship Approximation (-

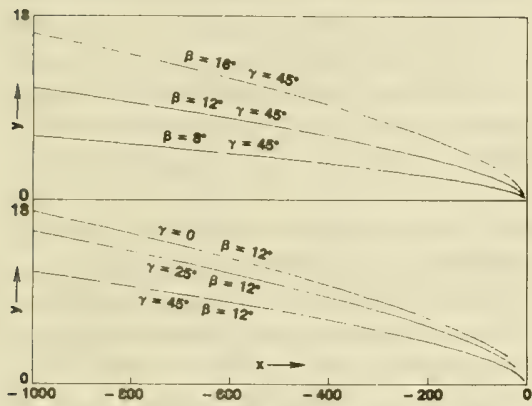

Fig. 22 - Lines Along Which the Steepness of the Divergent Waves is Equal to $1 / 15$ for a Simple Ship Bow Form with $\beta=8^{\circ}, 12^{\circ}, 16^{\circ}$ and $\gamma=45^{\circ}$ (Top) and $y=0,25^{\circ}, 45^{\circ}$ and $\beta=12^{\circ}$ (Bottom) in the Zeroth-

Order Slender-Ship Approximation

by the Michell thin-ship approximation $K_{M}(t)$ and the zeroth-order slender-ship approximation $\mathrm{K}_{0}(\mathrm{t})$. This figure strongly suggests the need for performing additional calculations based on a more realistic mathematical model than the simple thin-ship and slender-ship approximations used in this study.

Finally, the effect of the entrance angle $\beta$ and of the flare angle $y$ on the steepness of the divergent waves is illustrated in figure 22. Specifically, this figure depicts the lines along which the steepness of the divergent waves, as predicted by the slender-ship approximation $\mathrm{K}_{0}(\mathrm{t})$, is equal to $l / 15$ for $\beta=8^{\circ}, 12^{\circ}, 16^{\circ}$ and $\gamma=45^{\circ}$, in the top half of the figure, and for $\gamma=0,25^{\circ}, 45^{\circ}$ and $\beta=12^{\circ}$ in the bottom half of the figure. This figure shows that the short divergent waves in the Kelvin wake become steeper as the entrance angle $\beta$ increases and as the flare angle $\gamma$ decreases. More generally, figure 22 shows that the short divergent waves in the Kelvin wake are strongly influenced by the hull shape, and it therefore suggests the need for performing additional calculations in which systematic variations in hull shape are considered.

\section{SUMMARY OF RESULTS AND CONCLUSIONS}

Asymptotic expressions for determining the velocity potential and its derivatives at a sufficiently-large distance behind a ship advancing at constant speed in calm water are given by equations (23), (23a-e), (24a,b), (25a-d), (20ae) and (26a-d). The far-field asymptotic approximation (23) is uniformly valid in the vicinity of the track of the ship $a=0$ if condition (27) is satisfied. For the simple bow shape considered in this study, condition (27) is satisfied for $\mathrm{k}=0$ and 1 , corresponding to the potential $\phi$ and the free-surface elevation $\phi_{x}$, when the far-field wave-amplitude function $K(t)$ is approximated by the Michell thin-ship approximation $\mathrm{K}_{\mathrm{M}}(\mathrm{t})$ or the zeroth-order slender-ship approximation $\mathrm{K}_{0}(\mathrm{t})$. However, condition (27) is not satisfied for $k \geqslant 4$ and $k \geqslant 2$ for the approximations $\mathrm{K}_{\mathrm{M}}$ and $\mathrm{K}_{0}$, respectively.

The asymptotic approximations used in this study provide simple explicit analytical expressions for determining the velocity potential and its derivatives for large values of $-\mathrm{Xg} / \mathrm{U}^{2}$, that is in the far field, in terms of the far-field wave-amplitude function. However, for small and intermediate values of $\mathrm{Xg} / \mathrm{U}^{2}$, these asymptotic approximations are not useful, and the integrals (18) must be evaluated numerically. For intermediate values of $\mathrm{Xg} / \mathrm{U}^{2}$, the exponential function $\mathrm{E}_{+}(\mathrm{t} ; \overline{\mathrm{x}})+\mathrm{E}_{-}(\mathrm{t} ; \overline{\mathrm{x}})$ in the integrands of the integrals $(1),(3 a, b)$ and $(5 a, b)$ oscillates fairly rapidly, as may be seen from figure 1. Accurate and efficient integration rules suited to oscillatory integrands of the type depicted in figure 1 must be used. For small values of $\mathrm{Xg} / \mathrm{U}^{2}$, on the other hand, the oscillations of the exponential function $E_{+}(t ; \bar{x})+E_{-}(t ; \bar{x})$ are not significantly more rapid than the oscillations of the farfield wave-amplitude function $\mathrm{K}(\mathrm{t})$ which also appears in the integrands of the wave integrals (18), so that a different integration rule is required.

The amplitude, $a_{+}$, of the divergent waves in the Kelvin wake vanishes at the track of the ship if condition (37) is satisfied. However, it is well known that the 
wavelength, $\lambda_{+}$, of the divergent waves also vanishes at the track of the ship, as may be seen from figures 6 and

7. Therefore, the divergent waves can theoretically become infinitely steep at the track of the ship. More precisely, the steepness, $s_{+}=a_{+} / \lambda_{+}$, of the divergent waves is unbounded at the track of the ship if condition (36) is satisfied. Both conditions (37) and (36) can be satisfied simultaneously if condition (39) is satisfied, where the farfield wave-amplitude function is of order $1 / t^{\mu}$ as $t \rightarrow \infty$. Conditions (37) and (36), and consequently also condition (39), are satisfied in the cases of the thin-ship and the slender-ship approximations $K_{M}$ and $K_{0}$ for the simple ship form considered in the study, as may be verified from figures 15 and 16 where $\mathrm{a}_{+}(\alpha) \rightarrow 0$ and $\mathrm{s}_{+}(\alpha) \rightarrow \infty$ as $\alpha \rightarrow 0$.

Infinitely-steep water waves cannot exist in reality. Indeed, there exists a theoretical upper bound for the steepness of water waves in deep water, which is approximately equal to $1 / 7$. Condition (39) thus suggests that no divergent waves can exist within a certain region in the vicinity of the track of the ship, and that the Kelvin wake contains three distinct regions: (i) an inner region adjacent to the track of the ship where only transverse waves can exist, (ii) an outer region where both transverse and divergent waves are present, and (iii) an intermediate region at the boundary between the inner and outer regions where steep short divergent waves, as well as transverse waves, can be found.

Surface-tension and nonlinear effects have been ignored in the analysis presented in this study. This linear no-surface-tension analysis predicts extremely short and steep waves in the vicinity of the track of the ship. Both surface-tension and nonlinear effects therefore are liable to be significant, and these effects should be taken into account. A linear analysis including surface-tension effects should be performed first, since it is evident from the results obtained in the present study and from the brief description of the effects of surface tension upon the Kelvin wake given in Lamb [9, pp. 468-470] and Wehausen and Laitone [10, pp. 636-637] that the system of divergent waves in the vicinity of the track of the ship is likely to be profoundly affected by surface tension.

It was previously found by Scragg [5] that, for a ship bow form with a large flare angle, the zeroth-order slender-ship approximation $\mathrm{K}_{0}(\mathrm{t})$ predicts a sharp peak in the value of the amplitude of the divergent waves at a value of $\alpha$ equal to approximately half the entrance angle $\beta$. This finding of Scragg has been verified in this study, as may be seen from figures 15,16 and 18 . Furthermore, the magnitude of the stcepness of the divergent waves has been found to increase very rapidly as the Froude number decreases below a certain threshold value, as is shown in figure 19.

The line along which the steepness $s_{+}(a)$ of the divergent waves has a peak and the lines along which $s_{+}(\alpha)$ takes the large values $1 / 7,1 / 15$ and $1 / 20$ have been determined, for a simple ship form, on the basis of both the zeroth-order slender-ship approximation $K_{0}(t)$ and the Michell thin-ship approximation $K_{M}(t)$. Figure 20 , corresponding to the slender-ship approximation $\mathrm{K}_{0}(\mathrm{t})$, shows that these lines are well inside the Kelvin angle, and that the large-steepness lines are much closer to the track of the ship than the line corresponding to the peak in the steepness of the divergent waves.

The lines, depicted in figures 20 and 21 , along which the steepness of the divergent waves takes large constant values are independent of the value of the Froude number, but they strongly depend on the hull shape, as may be seen from figure 22 where "constantsteepness lines" corresponding to several values of the entrance angle $\beta$ and of the flare angle $\gamma$ are depicted. This figure shows that the short divergent waves in the Kelvin wake become steeper as the entrance angle increases and/or as the flare angle decreases.

It was found that the lines, along which the steepness of the divergent waves takes large constant values, predicted by the slender-ship approximation $\mathrm{K}_{0}(\mathrm{t})$ and the thin-ship approximation $\mathrm{K}_{\mathrm{M}}(\mathrm{t})$ are quite different from one another, as may be seen from figure 21 . This figure therefore indicates the need for performing additional calculations based on a more realistic mathematical model than the simple thin-ship and slendership approximations used in this study. These two approximations correspond to simple special cases of the Neumann-Kelvin theory, which should then be used. In particular, it would be useful to determine whether this more realistic theory predicts that the steepness of the divergent waves in the Kelvin wake exhibits a peak (or several peaks), as was found by using the slender-ship approximation $\mathrm{K}_{0}(\mathrm{t})$ for a ship bow form with large flare angle. Figure 21 specifically demonstrates the importance of obtaining accurate predictions of the far-field wave- 
amplitude function $K(t)$ for large values of $t$. Indeed, the short divergent waves in the vicinity of the track of the ship, that is for small values of $\alpha$, are associated with tise value of the function $K(t)$ for large values of $t$, as may be seen from equations (23), (24a), (25a) and (12). Precise knowledge of the asymptotic behavior of the function $\mathrm{K}(\mathrm{t})$ as $t \rightarrow \infty$ therefore is critical.

The behavior of the function $\mathrm{K}(\mathrm{t})$ as $\mathrm{t} \rightarrow \infty$ has been determined analytically in this study for the simple case of the thin-ship and slender-ship approximations $K_{M}(t)$ and $K_{0}(t)$ for an idealized ship bow form. More precisely, the asymptotic behavior of the functions $\mathrm{K}_{\mathrm{M}}(\mathrm{t})$ and $K_{0}(t)$ for the simple ship bow shape considered in this study is specified by equations $(45)$ and $(67 a, b)$. These asymptotic approximations show that we have $\left|\mathrm{K}_{\mathrm{M}}(\mathrm{t})\right|=$ $O\left(1 / t^{3}\right)$ and $\left|K_{0}(t)\right|=O\left(1 / t^{2}\right)$ as $t \rightarrow \infty$. They also show that the Michell thin-ship approximation $K_{M}(t)$, which corresponds to the thin-ship limit of the slender-ship approximation $\mathrm{K}_{0}(\mathrm{t})$, is not uniformly valid in the limit $\mathrm{t}$ $\rightarrow \infty$. It is possible to analytically determine the behavior of the far-field wave-amplitude function $K(t)$ associated with the Neumann-Kelvin theory for an arbitrary ship form, as is shown in [12]. Such an asymptotic approximation for the function $K(t)$ for large values of $t$ is useful because it provides an explicit analytical relationship between the hull form and the Froude number, on one hand, and the amplitude of the short divergent waves in the vicinity of the track of the ship, on the other hand.

\section{ACKNOWLEDGMENTS}

This study was funded by the Office of Naval Technology sponsored Exploratory Development Surface Ship Wake Detection Project at the David W. Taylor Naval Ship R\&D Center. The authors wish to thank Dr. Arthur Reed and Mr. Seth Hawkins for their interest in the study and for their useful comments.

\section{REFERENCES}

1. Fu, Lee-Lueng and Benjamin Holt, "Seasat Views Oceans and Sea Ice with Synthetic Aperture Radar", JPL Publication 81-120, 15 Feb 1982.

2. McDonough, Robert N., Barry E. Raff and Joyce L. Kerr, "Image Formation from Spaceborne Synthetic Aperture Radar Signals", Johns Hopkins APL Technical Digest, Vol. 6, No. 4, Oct-Dec 1985, pp. 300-312.

3. Swanson, Claude V., "Radar Observability of Ship Wakes", Applied Physics Technology Report No. I, May $1984,109 \mathrm{pp}$.

4. Case, K.M., et al, "Seasat Report", MITRE Corporation, Report JSR-83-203, March 1984.

5. Scragg, Carl A., "A Numerical Investigation of the Kelvin Wake Generated by a Destroyer Hull Form", Science Applications, Report No. SAI-83/1216, Oct 1983, pp. 46.

6. Cooper, A.L., "Interactions Between Ocean Surface Waves and Currents", Naval Research Laboratory, NRL Memorandum Report 5755, April 1986. pp. 18.

7. Noblesse, F., "A Slender-Ship Theory of Wave Resistance", Journal of Ship Research, Vol. 27, No. 1, March 1983, pp. 13-33.

8. Sharma, S.D., "Some Results Concerning the Wavemaking of a Thin Ship", Journal of Ship Research, Vol. 13, 1969, pp. 72-81.

9. Lamb, H., "Hydrodynamics", Dover Publications, New York, 1879, pp. 738.

10. Wehausen, J.V. and E.V. Laitone, "Surface Waves", in Encyclopedia of Physics, Springer-Verlag, Berlin, Vol. IX, 1960, pp. 446-778.

11. Ursell, F., “On Kelvin's Ship-Wave Pattern", Journal of Fluid Mechanics, Vol. 8, 1960, pp. 418-431.

12. Noblesse, F., "Analytical Approximations for Steady Ship Waves at Low Froude Numbers", 21st ATTC Resistance and Flow Committee, Washington, Aug. 1986. 

INITIAL DISTRIBUTION

Copies

1

3

1 CONR/Boston

1 CONR/Chicago

1 CONR/Pasadena

1 NAVPGSCOL

1 NROTC \& NAVADMINU

1 NAVWARCOL

$5 \quad$ NRL

1 Lib

45841

10
Copies

$\begin{array}{cl}1 & \text { NUSC NPT } \\ 1 & \text { NUSC NLONLAB } \\ 1 & \text { NAVSHIPYD BREM/Lib } \\ 1 & \text { NAVSHIPYD CHASN/Lib } \\ 1 & \text { NAVSHIPYD MARE/Lib } \\ 1 & \text { NAVSHIPYD NORVA/Lib } \\ 1 & \text { NAVSHIPYD PEARL/Lib } \\ 1 & \text { NAVSHIPYD PHILA } \\ 12 & \text { DTIC } \\ 1 & \text { AFFDL/FDDS/J. Olsen } \\ 2 & \text { AFFDL/FYS } \\ 1 & \text { Dale Cooley } \\ & \text { S.J. Pollock }\end{array}$

2

1

1

1

1

4

1 NASA/Sci \& Tech Info tity

1 NSF/Eng Div 
Univ of Bridgeport

Prof. E. Uram

Mech Eng Dept

5

3

1

1

1

1

2

1

1

4

$$
\begin{aligned}
& \text { State Univ of Iowa } \\
& \text { Iowa Inst of Hyd Research } \\
& 1 \text { L. Landweber } \\
& 1 \text { J. Kennedy } \\
& 1 \text { V.C. Patel } \\
& 1 \text { F. Stern }
\end{aligned}
$$

Univ of California, Berkeley

$$
\begin{array}{ll}
1 & \text { Lib } \\
1 & \text { J.R. Paulling } \\
1 & \text { J.V. Wehausen } \\
1 & \text { W. Webster } \\
1 & \text { R. Yeung }
\end{array}
$$

CA Inst of Tech

$$
\begin{array}{ll}
1 & \text { A.J. Acosta } \\
1 & \text { T.Y. Wu } \\
1 & \text { Lib }
\end{array}
$$

Lehigh Univ Fritz Lab Lib

Long Is land Univ Grad Dept of Marine Sci David Price

Delaware Univ/Math Dept

$$
\begin{aligned}
& \text { Univ of Maryland } \\
& 1 \text { Eng Lib } \\
& 1 \text { p } \\
& 1 \text { C.L. S } \\
& 1 \text { F. Buckley }
\end{aligned}
$$

MIT/Dept of Ocean Eng

1 J.R. Kerwin

1 J.N. Newman

1 P. Leehey

1 M. Abkowitz

1 A.T. Ippen/Hydro Lab

1 T.F. Ogilvie

Univ of Michigan/Dept/NAME

1 Lib

1 R. Beck

1 R.B. Couch

1 W. Vorus

1 T.E. Brockett

Univ of Minn/St. Anthony Falls

1 R. Arndt

1 C. Fare 11

1 J.M. Killen

1 F. Schiebe

1 J.M. Wetzel

City College, Wave Hill

1 W.J. Pierson, Jr.

1 A.S. Peters

1 J.J. Stoker

Univ of Notre Dame

A.F. Strandhagen

Penn State Univ

Applied Research Lab

SAI/Annapolis

1 N. Salvesen

1 C. von Kerczek 
Southwest Research Inst

1 H.N. Abramson

1 G.E. Transleben, Jr .

1 Applied Mech Review

4

$$
\begin{aligned}
& \text { Stanford Univ/Dept of Div Eng } \\
& 1 \text { R.L. Street } \\
& 1 \text { B. Perry } \\
& 2 \text { Dept of Aero and Astro/ } \\
& \text { J. Ashley } \\
& \text { M. Van Dyke }
\end{aligned}
$$

\section{Stanford Research Inst/Lib}

Stevens Inst of Tech/Davidson Lab 1 D. Savitsky

1 Lib

Utah State Univ/Co1 of Eng Roland W. Jeppson

Univ of Virginia/Aero Eng Dept

1 J.K. Haviland

1 Young Yoo

Webb Institute

1 Lib

1 L.W. Ward

Worcester Poly Inst/Alden

Research Lab

Woods Hole, Ocean Eng Dept

SNAME

Aerojet-General/W.C. Beckwith

Bethlehem Steel Sparrows Tech Mgr

Bolt, Beranek \& Newman, MA

Boeing Company/Aerospace Group

1 R.R. Barberr

1 W.S. Rowe

1 P.E. Rubbert

1 G.R. Saaris

CALSPAN, Inc. Applied Mech Dept
Flow Research, Inc.

\section{Eastern Research Group}

General Dynamics Corp.

1 Convair Aerospace Div A.M. Cunningham, Jr. Ms 2851

1 Electric Boat Div V.T. Boatwright, Jr.

Gibbs \& Cox, Inc. Tech Info Control Section

Grumman Aircraft Eng Corp W.P. Car1, Mgr/Grumman Marine

Tracor Hydronautics, Inc.

1 G. Miller

1 A. Goodman

Lockheed Aircraft Corp

Lockheed Missiles \& Space

1 R.L. Waid

1 R. Lcay

1 R. Perkins

1 R. Kramer

Marquadt Corp/F. Lane General Applied Sci Labs

Martin Marietta Corp/Rias

McDonne11-Douglas Corp/Douglas

Aircraft Company

1 Lib

1 T. Cebeci

Newport News Shipbuilding/Lib

Nielsen, NA Rockwel1

North American Rockwell

Los Angeles Div

J.R. Tulinius/Dept 056-015

Northrop Corp/Aircraft Div

1 J.T. Gallagher

1 J.R. Stevens 


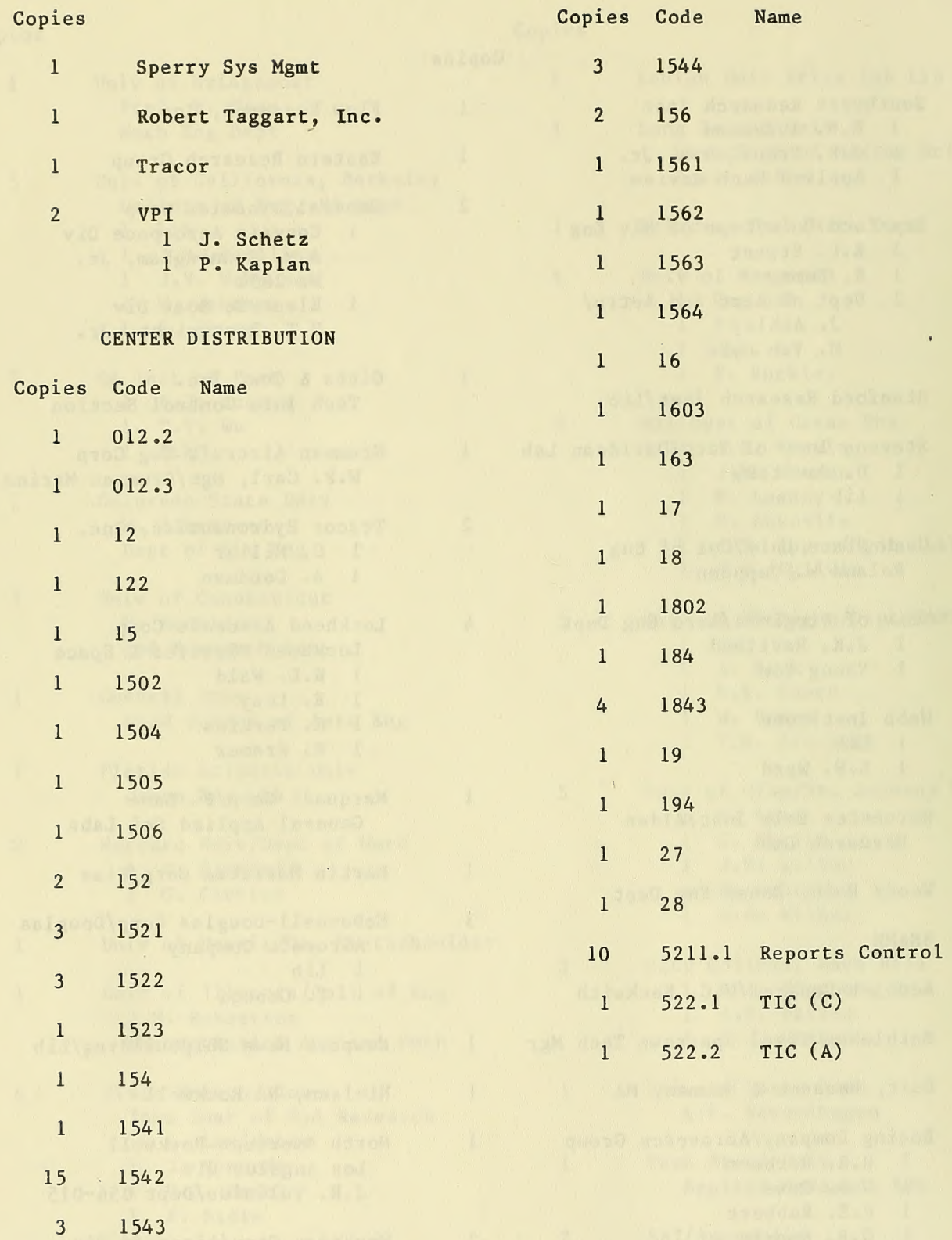




\section{DTNSRDC ISSUES THREE TYPES OF REPORTS:}

1. DTNSRDC reports, a formal series. contain information of permanent technical value. They carry a consecutive numerical identification regardless of their classification or the originating department.

2. Departmental reports, a semiformai series, contain information of a preliminary, temporary, or proprietary nature or of limited interest or significance. They carry a departmental alphanumerical identification.

3. Technical memoranda, an informal series, contain technical documentation of limited use and interest. They are primarily working papers intended for internal use. They carry an identifying number which indicates their type and the numerical code of the originating department. Any distribution outside DTNSRDC must be approved by the head of the originating department on a case-by-case basis. 


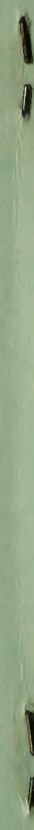

